\title{
ERROR BOUNDS FOR EXPONENTIALLY IMPROVED ASYMPTOTIC SOLUTIONS OF ORDINARY DIFFERENTIAL EQUATIONS HAVING IRREGULAR SINGULARITIES OF RANK ONE
}

\author{
T. M. Dunster
}

\begin{abstract}
Solutions of general homogeneous linear second-order differential equations having an irregular singularity, of unit rank, at infinity are examined. The classical Poincaré expansions for such solutions generally are valid in a sector for which the argument of the independent variable $z$ has the range $3 \pi-\delta$ where $\delta$ denotes an arbitrary positive constant. In this paper, $n$ terms, say, are taken in the Poincaré expansion; the remainder is expressed in the form $h_{n}(z)+\varepsilon_{n}(z)$, and an explicit expansion involving the generalised exponential integral then is derived for $h_{n}(z)$. By the method of successive approximations, explicit error bounds are derived for $\left|\varepsilon_{n}(z)\right|$. Then it is shown from the error bounds that when $n=|z|+\alpha$ where $\alpha$ is bounded and $|z| \rightarrow \infty, \varepsilon_{n}(z)$ is asymptotically smaller than $h_{n}(z)$ in a sector with a range of $\arg (z)$ which is larger than $4 \pi$. In addition to being valid in a larger sector than the corresponding Poincaré expansions, the expansions provide greater accuracy, and give a smooth interpretation of the Stokes phenomenon. The present results provide explicit and realistic error bounds for general exponentially-improved asymptotic solutions of differential equations.
\end{abstract}

\section{Introduction}

In this paper, we shall investigate solutions of the general homogeneous linear secondorder differential equation of the form

$$
\frac{d^{2} w}{d z^{2}}+f(z) \frac{d w}{d z}+g(z) w=0 .
$$

In particular, we are interested in the case where the equation has an irregular singularity, of unit rank, at infinity. The general theory for such a problem is well-known; for full details see, for example, [8, Chap.7, §§1-2].

The notation we adopt is as follows. A positive number $a$ and coefficients $\left\{f_{s}\right\}_{s=0}^{\infty}$ and $\left.\left\{g_{s}\right\}_{s=0}^{\infty}\right\}$ are such that for $|z|>a$, the following series converge

$$
f(z)=\sum_{s=0}^{\infty} \frac{f_{s}}{z^{s}}, \quad g(z)=\sum_{s=0}^{\infty} \frac{g_{s}}{z^{s}} .
$$

Since the singularity at infinity is not regular, it follows that

$$
\left|f_{0}\right|+\left|g_{0}\right|+\left|g_{1}\right| \neq 0 \text {. }
$$

Received April 18, 1995, revised November 10, 1995.

1991 Mathematics Subject Classification. Primary: 34E05, Secondary: 41A30, 41A60, 30E10, $30 \mathrm{E} 15$.

Key words and phrases: asymptotic expansions, ordinary differential equations, approximation in the complex domain. 
The equation has unique solutions $w_{1}(z)$ and $w_{2}(z)$ satisfying

$$
w_{j}(z) \sim e^{\lambda_{j} z} z^{\mu_{j}} \sum_{s=0}^{\infty} \frac{a_{s, j}}{z^{s}} \quad\left(z \rightarrow \infty,\left|\arg \left\{(-1)^{j}\left(\lambda_{1}-\lambda_{2}\right) z\right\}\right| \leq \frac{3}{2} \pi-\delta\right),
$$

for some constants $\left\{a_{s, j}\right\}_{s=0}^{\infty}$ which can be determined recursively in terms of the coefficients appearing in (1.2), (see, for example, [8, p.230]). Here, and throughout, $\delta$ denotes an arbitrary small positive constant.

Without loss of generality, we shall assume that $a_{0,1}=a_{0,2}=1$. The constants $\lambda_{j}$ are the roots of the quadratic equation

$$
\lambda^{2}+f_{0} \lambda+g_{0}=0,
$$

and without loss of generality (see [7]), we shall assume that they are distinct, satisfying

$$
\lambda_{2}-\lambda_{1}=1
$$

Thus, in the notation of $[8$, Chap. 7$]$, we have

$$
\begin{aligned}
\rho & =\frac{1}{2}, \quad \sigma=\frac{1}{2} f_{0} f_{1}-g_{1}, \\
\lambda_{1} & =-\frac{1}{2}-\frac{1}{2} f_{0}, \quad \lambda_{2}=\frac{1}{2}-\frac{1}{2} f_{0}, \\
\mu_{1} & =-\sigma-\frac{1}{2} f_{1}, \quad \mu_{2}=\sigma-\frac{1}{2} f_{1} .
\end{aligned}
$$

If expansions of the form (1.4) are truncated after an optimal number ( $n$ say) of terms, it is known for a wide class of functions that the remainder can be reexpanded in terms of generalised exponential integrals. These integrals in turn can be represented in terms of the complementary error function (see [2], [5] and [9]), and as a result, a smooth interpretation of the Stokes phenomenon is provided. The two other major advantages of this technique are greater attainable accuracy and a larger sector of validity. In particular, the extended sectors of validity are

$$
0 \leq\left|\arg \left(z e^{-\pi(j-1) i}\right)\right| \leq \frac{5}{2} \pi-\delta
$$

which should be compared with (1.4) and (1.6). Another important advantage of being valid in these larger sectors is that it can allow the determination of the connection coefficients between any three solutions of (1.1).

The smoothing of Stokes discontinuities, for certain functions having integral representations, first was achieved by Berry [2] in 1989, and later for solutions of differential equations in [3]. Subsequently, the technique was put on a more rigorous foundation, and generalised to solutions of differential equations by a number of authors, including Paris [11], McLeod [6], Olver [10], and Olde Daalhuis and Olver [7]. The scope of the latter two papers is very close to that of the present paper. In [10], a direct differential equation approach was used to obtain exponentially-improved re-expansions for the asymptotic series of the confluent hypergeometric function. In [7], similar results to those in [10] were obtained for the general equation (1.1), and in both papers, it was assumed that certain connection coefficients (the so-called Stokes multipliers) were known. In [7], Stieltjes-type representations were constructed for the remainder terms, and from these, the desired re-expansions in terms of generalised exponential integrals were obtained, together with order estimates (in certain restricted sectors) for the error terms; by use of appropriate connection formulae, these estimates were 
used to show that the error terms were asymptotically smaller than the expansions throughout the extended sectors (1.10).

In this paper, we take an approach different from [7]. After truncating the Poincaré expansion after $n$ terms, we obtain the re-expansion involving generalised exponential integrals via an inhomogeneous differential equation satisfied by the remainder term. This expansion involves certain coefficients which can be determined directly from the coefficients in (1.2) and (1.4), and no use is required of connection formulae. This has the potential advantage of facilitating calculation of the Stokes multipliers. It should be remarked, however, that since the Stokes multipliers appear directly in the re-expansions in [7] and [10], they enjoy the property of giving a particularly elegant interpretation of the smoothing of Stokes discontinuities.

The main significance of this paper is the derivation of realistic and explicit error bounds. Previously, the only explicit error bounds given for this class of problem was for a specific function, namely the generalised exponential integral by Dunster [5]. In this, error bounds were derived using an asymptotic theory for a class of ordinary differential equations having almost coalescent turning points [4].

The plan of this paper is as follows. In $\S 2$, we give error bounds which are uniformly valid for $0 \leq\left|\arg \left(z e^{-\pi(j-1) i}\right)\right| \leq 2 \pi$, initially focusing on this smaller sector rather than the full sector of asymptotic validity (1.10) so as to simplify the error analysis. In $\S 3$, we give details of the proof of the derivation of these bounds, which uses the technique of successive approximations. It is seen from the bounds that the relative error in this case is $O\left(z^{-1}\right)$ as $z \rightarrow \infty$. In $\S 4$, we generalise the results of $\S \S 2-3$ to give exponentially-improved expansions with an improved relative error term of $O\left(z^{-m}\right)$ as $z \rightarrow \infty$ where $m$ is a prescribed fixed positive integer. Again these are uniformly valid for $0 \leq\left|\arg \left(z e^{-\pi(j-1) i}\right)\right| \leq 2 \pi$.

In $\S 5$, we give brief details on the extension of the error analysis to sectors which, in conjunction with the results of $\S 4$, give explicit error bounds for the sectors (1.10). In fact, the results of $\S \S 2-4$, together with appropriate connection formulae, are sufficient to provide error bounds for exponentially-improved asymptotic expansions for all ranges of $\arg (z)$; however, the importance of $\S 5$ is to both demonstrate that the sectors of validity of $\S \S 2-4$ are not maximal and to show clearly how the analysis breaks down $\operatorname{as} \arg (z)$ approaches the extreme values of (1.10). In $\S 6$, we examine in more detail the asymptotic nature of the error bounds, which involve so-called weight functions. In particular, we show that when $n=|z|+O(1)$ each error term is asymptotically smaller than the corresponding approximant throughout a sector which is larger than $0 \leq\left|\arg \left(z e^{-\pi(j-1) i}\right)\right| \leq 2 \pi$. Finally, in $\S 7$, we give a numerical example on the calculation of certain constants which appear.

\section{Smoothing of Stokes discontinuities: the first approximation}

Let us consider $w_{1}(z)$. From our definitions in $\S 1$, we know that

$$
w_{1}(z) \sim e^{\lambda_{1} z} z^{\mu_{1}} \sum_{s=0}^{\infty} \frac{a_{s, 1}}{z^{s}} \quad\left(z \rightarrow \infty,|\arg (z)| \leq \frac{3}{2} \pi-\delta\right) .
$$

Following [7], our aim is to approximate this solution, for large $z$, in a domain which is larger than that for which (2.1) is valid. To do this, choose $n \in \mathbf{N}$, and express the solution in the form

$$
w_{1}(z)=e^{\lambda_{1} z} z^{\mu_{1}} \sum_{s=0}^{n-1} \frac{a_{s, 1}}{z^{s}}+h_{n, 1}(z)
$$


Substitution into (1.1) yields the inhomogeneous equation

$$
h_{n, 1}^{\prime \prime}(z)+f(z) h_{n, 1}^{\prime}(z)+g(z) h_{n, 1}(z)=-e^{\lambda_{1} z} z^{\mu_{1}} r_{n, 1}(z)
$$

where

$$
r_{n, 1}(z)=-\frac{n a_{n, 1}}{z^{n+1}}+\frac{\hat{r}_{n, 1}(z)}{z^{n+2}}
$$

with

$$
\hat{r}_{n, 1}(z)=\sum_{s=0}^{n-1} a_{s, 1}\left\{\left(\mu_{1}-s\right) \hat{F}_{n+1-s}(z)+\lambda_{1} \hat{F}_{n+2-s}(z)+\hat{G}_{n+2-s}(z)\right\}
$$

and

$$
\hat{F}_{n}(z)=\sum_{s=0}^{\infty} \frac{f_{s+n}}{z^{s}}, \quad \hat{G}_{n}(z)=\sum_{s=0}^{\infty} \frac{g_{s+n}}{z^{s}} .
$$

The remainder term $h_{n, 1}(z)$ is the unique solution of (2.3), which is $O\left(e^{\lambda_{1} z} z^{\mu_{1}-n}\right)$ as $z \rightarrow+\infty(\arg (z)=0)$.

We wish to use variation of parameters to re-express (2.3) as a Volterra integral equation. To do so, we seek a homogeneous differential equation which is "close" to that on the left-hand side of (2.3), having explicit elementary solutions. The following lemma is what we require (cf. [8, Chap.7, §12]).

Lemma 2.1. The elementary functions $e^{\lambda_{1} z} z^{\mu_{1}}$ and $e^{\lambda_{2} z} z^{\mu_{2}}$ are independent solutions of the differential equation

$$
\frac{d^{2} w}{d z^{2}}+\tilde{f}(z) \frac{d w}{d z}+\tilde{g}(z) w=0
$$

where

$$
\tilde{f}(z)=f_{0}+\frac{f_{1}+1}{z}-\frac{1}{z+2 \sigma}
$$

and

$$
\tilde{g}(z)=g_{0}+\frac{2 \sigma g_{1}-f_{0} g_{1}+2 g_{0} f_{1}}{2 \sigma z}+\frac{f_{1}-2 \sigma f_{0}}{4 \sigma(z+2 \sigma)}+\frac{f_{1}^{2}-4 \sigma^{2}}{4 z^{2}} .
$$

This lemma obviously can be verified by direct substitution. The equation (2.7) was derived by first observing that $\omega(z) e^{\lambda_{j} z} z^{\mu_{j}}=e^{ \pm \zeta}(j=1,2)$ where $\omega(z)=$ $z^{f_{1} / 2} \exp \left\{\frac{1}{2} f_{0} z\right\}$ and $\zeta=-\frac{1}{2} z-\sigma \ln (z)$. Thus, $\omega(z) e^{\lambda_{j} z} z^{\mu_{j}}(j=1,2)$ both satisfy the equation $d^{2} y / d \zeta^{2}-y=0$ : on substituting $y=\omega w$ and returning to the original independent variable $z$, we obtain (2.7).

Note that for large $z$,

$$
\tilde{f}(z)=f_{0}+\frac{f_{1}}{z}+\frac{2 \sigma}{z^{2}}+O\left(\frac{1}{z^{3}}\right)
$$

and

$$
\tilde{g}(z)=g_{0}+\frac{g_{1}}{z}+\frac{g_{0} f_{1}\left(2-f_{1}\right)+g_{1}\left(f_{0} f_{1}-f_{0}-g_{1}\right)}{z^{2}}+O\left(\frac{1}{z^{3}}\right) .
$$

Thus, these functions differ, respectively, from $f(z)$ and $g(z)$ by $O\left(z^{-2}\right)$.

Now we rewrite (2.3) in the form

$$
\begin{aligned}
h_{n, 1}^{\prime \prime}(z)+ & \tilde{f}(z) h_{n, 1}^{\prime}(z)+\tilde{g}(z) h_{n, 1}(z) \\
& =-e^{\lambda_{1} z} z^{\mu_{1}} r_{n, 1}(z)+\{\tilde{g}(z)-g(z)\} h_{n, 1}(z)+\{\tilde{f}(z)-f(z)\} h_{n, 1}^{\prime}(z) .
\end{aligned}
$$


On recalling that $h_{n, 1}(z)=O\left(e^{\lambda_{1} z} z^{\mu_{1}-n}\right)$ as $z \rightarrow+\infty$, we deduce that

$$
h_{n, 1}(z)=\int_{z}^{\infty} K(z, t)\left\{-e^{\lambda_{1} t} t^{\mu_{1}} \chi(t) r_{n, 1}(t)+\frac{\tilde{G}(t)}{t^{2}} h_{n, 1}+\frac{\tilde{F}(t)}{t^{2}} h_{n, 1}^{\prime}(t)\right\} d t
$$

where

$$
\chi(t)=\left(1-\frac{\mu_{1}-\mu_{2}}{t}\right)^{-1}
$$

and

$$
K(z, t)=e^{\lambda_{1} z} z^{\mu_{1}} e^{-\lambda_{1} t} t^{-\mu_{1}}-e^{\lambda_{2} z} z^{\mu_{2}} e^{-\lambda_{2} t} t^{\mu_{2}} .
$$

In addition, we have defined

$$
\tilde{F}(t)=t^{2} \chi(t)\{\tilde{f}(t)-f(t)\}, \quad \tilde{G}(t)=t^{2} \chi(t)\{\tilde{g}(t)-g(t)\},
$$

both of which are $O(1)$ as $t \rightarrow \infty$.

We shall approximate $h_{n, 1}(z)$ by $h_{n, 1}^{(1)}(z)$, which is the function given by the righthand side of (2.13) with $h_{n, 1}(t)$ and $h_{n, 1}^{\prime}(t)$ neglected. Thus, define

$$
h_{n, 1}^{(1)}(z)=e^{\lambda_{2} z} z^{\mu_{2}} \int_{z}^{\infty} e^{-t} t^{\mu_{1}-\mu_{2}} \chi(t) r_{n, 1}(t) d t-e^{\lambda_{1} z} z^{\mu_{1}} \int_{z}^{\infty} \chi(t) r_{n, 1}(t) d t .
$$

To estimate, and indeed bound, the error in this approximation, we shall use the wellknown technique of successive approximations. To this end, we define for $s=1,2, \ldots$

$$
h_{n, 1}^{(s, 1)}(z)=\int_{z}^{\infty} K(z, t)\left\{\frac{\tilde{G}(t)}{t^{2}} h_{n, 1}^{(s-1,1)}(t)+\frac{\tilde{F}(t)}{t^{2}} h_{n, 1}^{(s-1,1)^{\prime}}(t)\right\} d t
$$

with the specification that $h_{n, 1}^{(0,1)}(t)=h_{n, 1}^{(1)}(t)$. In all the integrals above, the path of integration runs from $t=z$ to $t=+\infty($ with $\arg (t)=0)$ such that $|\arg (t)| \leq|\arg (z)|$ and $t$ lies outside a sufficiently large disk centred at the origin (thus avoiding all singularities of the respective integrands).

Let us return to the approximant defined by (2.17). By integration by parts, it can be expressed as

$$
h_{n, 1}^{(1)}(z)=e^{\lambda_{2} z} z^{\mu_{2}} \int_{z}^{\infty} e^{-t} t^{\mu_{1}-\mu_{2}} R_{n, 1}(t) d t
$$

where

$$
R_{n, 1}(t)=-\left\{1-\frac{\mu_{1}-\mu_{2}}{t}\right\} \int_{t}^{\infty} \chi(s) r_{n, 1}(s) d s .
$$

The function $R_{n, 1}(t)$ has the convergent expansion $(|t|>a)$

$$
R_{n, 1}(t)=\frac{a_{n, 1}}{t^{n}}+\sum_{k=1}^{\infty} \frac{\alpha_{n, 1}(k)}{t^{n+k}}
$$

with readily computed coefficients. It is worth observing that if $a_{s, 1}$ has a factorial growth as $s \rightarrow \infty$, then for large $n, \alpha_{n, 1}(k)=O\left(a_{n-1,1}\right)=o\left(a_{n, 1}\right)$ for each $k$. In general, this can be shown to be true (see for example [1] and [7]).

By expanding the integrand in (2.19), we find that the approximant $h_{n, 1}^{(1)}(z)$ can be expressed in terms of the generalised exponential integral, which is defined for $|\arg (z)|<\frac{1}{2} \pi$ by

$$
E_{p}(z)=\int_{1}^{\infty} \frac{e^{-z t}}{t^{p}} d t
$$


and by analytic continuation elsewhere. Then, from uniform absolute convergence, we interchange summation and integration, and as a result, we find that

$$
h_{n, 1}^{(1)}(z)=a_{n, 1} e^{\lambda_{2} z} z^{\mu_{1}-n+1} E_{n-\mu_{1}+\mu_{2}}(z)+e^{\lambda_{2} z} \sum_{k=1}^{\infty} \alpha_{n, 1}(k) z^{\mu_{1}-n-k+1} E_{n+k-\mu_{1}+\mu_{2}}(z) .
$$

To understand the asymptotic nature of this function, we wish to express the righthand side of (2.23) in terms of $E_{n-\mu_{1}+\mu_{2}}(z)$ alone. This can be done by employing the well-known expansion (see, for example, $[5, \S 4]$ )

$$
z^{-k} E_{p+k}(z)=(-1)^{k} \frac{\Gamma(p)}{\Gamma(p+k)} E_{p}(z)+\frac{e^{-z}}{z} \sum_{s=0}^{k-1}(-1)^{s+k+1} \frac{\Gamma(p+s)}{\Gamma(p+k) z^{s}} .
$$

Thus, assuming convergence of the following sums for a given set of coefficients $\tilde{\alpha_{k}}$ and large enough $|z|$, which is the case above, we have in general

$$
\begin{aligned}
\sum_{k=1}^{\infty} \tilde{\alpha_{k}} z^{-k} E_{p+k}(z)= & E_{p}(z) \sum_{k=1}^{\infty}(-1)^{k} \tilde{\alpha_{k}} \frac{\Gamma(p)}{\Gamma(p+k)} \\
& -\frac{e^{-z}}{z} \sum_{s=0}^{\infty}(-1)^{s} \frac{\Gamma(p+s)}{z^{s}} \sum_{k=s+1}^{\infty} \frac{(-1)^{k} \tilde{\alpha_{k}}}{\Gamma(p+k)} .
\end{aligned}
$$

Identifying (2.23) with (2.25), we set $p=n-\mu_{1}+\mu_{1}$ and $\tilde{\alpha_{k}}=\alpha_{n, 1}(k)$, thus obtaining the desired form

$$
h_{n, 1}^{(1)}(z)=a_{n, 1} e^{\lambda_{2} z} z^{\mu_{1}-n+1}\left[E_{n-\mu_{1}+\mu_{2}}(z)\left(1+\beta_{n, 1}^{(1)}(0)\right)-\frac{e^{-z}}{z} \sum_{s=0}^{\infty} \frac{\beta_{n, 1}^{(1)}(s)}{z^{s}}\right]
$$

where

$$
\beta_{n, 1}^{(1)}(s)=(-1)^{s} \sum_{k=s+1}^{\infty}(-1)^{k} \frac{\alpha_{n, 1}(k)}{a_{n, 1}} \frac{\Gamma\left(n+s-\mu_{1}+\mu_{2}\right)}{\Gamma\left(n+k-\mu_{1}+\mu_{2}\right)} \quad(s=0,1,2, \cdots) .
$$

For large $|z|$ with $|\arg (z)| \leq 3 \pi-\delta$ and $n=|z|+O(1),(2.26)$ can be reduced to

$$
h_{n, 1}^{(1)}(z)=a_{n, 1} e^{\lambda_{2} z} z^{\mu_{1}-n+1} E_{n-\mu_{1}+\mu_{2}}(z)\left[1+O\left(\frac{a_{n-1,1}}{n a_{n, 1}}\right)\right],
$$

except near the zeroes of the generalised exponential integral (see the comment below Eq. (2.21)). Moreover, the $O$-term is readily computed explicitly. In deriving (2.28), we have used the asymptotic form [5, Theorem 2.1] for $z \rightarrow \infty$ ( $n$ small or large)

$$
E_{n-\mu_{1}+\mu_{2}}(z) \sim \frac{e^{-z}}{z+n-\mu_{1}+\mu_{2}} \quad(|\arg (z)| \leq \pi-\delta),
$$

in conjunction with both the analytic continuation formula

$$
E_{p}\left(z e^{2 \pi i}\right)=\frac{2 \pi i}{\Gamma(p)} e^{p \pi i} z^{p-1}+E_{p}(z)
$$

and Stirling's formula for the gamma function when $n$ is large; in addition, we have used the fact that $\left|E_{n-\mu_{1}+\mu_{2}}(z)\right|^{-1}=O\left(n e^{-n}\right)$ in the vicinity of $z=n e^{\pi i}[5$, Theorem $3.1]$.

We shall bound each $h_{n, 1}^{(s)}(z)$ in turn in terms of a weight function $\mathcal{E}_{n, 1}(z)$ defined as follows. It will be defined as the supremum of a certain function over a region $\mathcal{D}(z)$ 
in the plane of a dummy complex variable $u$. To define this domain, first we introduce two regions in the $u$-plane (for a fixed nonzero $z$ ) by

$$
\begin{gathered}
\mathcal{D}_{1}^{+}(z)=\{u: 0 \leq \arg (u) \leq \arg (z),|u| \geq|z|, \operatorname{Re}(u) \geq \operatorname{Re}(z)\} \quad(0 \leq \arg (z) \leq \pi), \\
\mathcal{D}_{2}^{+}(z)=\{u: \pi<\arg (u) \leq \arg (z),|u|=|z|\} \quad(\pi<\arg (z) \leq 2 \pi) .
\end{gathered}
$$

Then we define, for $0 \leq \arg (z) \leq 2 \pi$,

$$
\mathcal{D}^{+}(z)=\left\{\begin{array}{ll}
\mathcal{D}_{1}^{+}(z) & (0 \leq \arg (z) \leq \pi) \\
\mathcal{D}_{1}^{+}\left(|z| e^{\pi i}\right) \cup \mathcal{D}_{2}^{+}(z) & (\pi<\arg (z) \leq 2 \pi)
\end{array} ;\right.
$$

see Figure 2.1. Next, again for $0 \leq \arg (z) \leq 2 \pi$, let $\mathcal{D}^{-}(z)$ be the region on the sheet $-2 \pi \leq \arg (u) \leq 0$ which is conjugate to $\mathcal{D}^{+}(z)$. Then, we define

$$
\mathcal{D}(z)=\mathcal{D}^{+}(z) \cup \mathcal{D}^{-}(z) \quad(0 \leq \arg (z) \leq 2 \pi)
$$

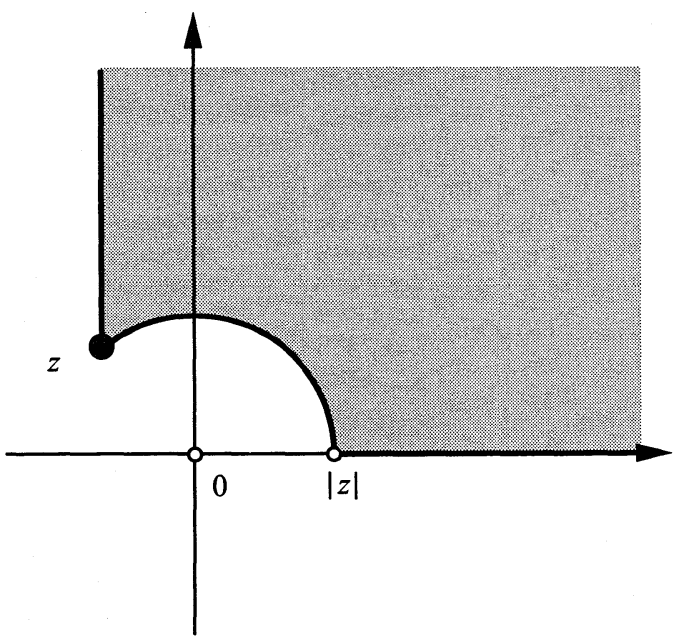

(a)

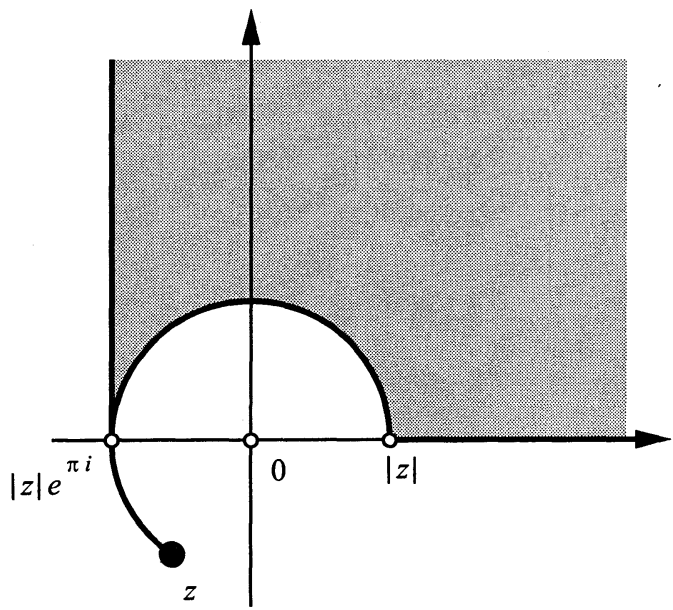

(b)

FiguRE 2.1. $u$-plane: domain $\mathcal{D}^{+}(z)$ (shaded) for: (a) $0<\arg (z)<\pi$, (b) $\pi<\arg (z)<2 \pi$. 
and

$$
\mathcal{D}(z)=\mathcal{D}(\bar{z}) \quad(-2 \pi \leq \arg (z)<0)
$$

where, in the latter, it is understood that $\arg (\bar{z})=-\arg (z)$ for $-2 \pi \leq \arg (z)<0$.

Our first observation is that $\mathcal{D}\left(\rho e^{i \theta_{1}}\right) \subset \mathcal{D}\left(\rho e^{i \theta_{2}}\right)$ when $\rho>0$ and $0 \leq \theta_{1}<\theta_{2} \leq 2 \pi$ or $-2 \pi \leq \theta_{2}<\theta_{1} \leq 0$. The principal motivating reason for the particular definition (2.31)-(2.35) is given in $\S 3$ (in the paragraph after (3.4)).

Having given the domain, we choose the smallest nonnegative integer $q$ such that

$$
q \geq\left|\operatorname{Re}\left(\mu_{1}-\mu_{2}\right)\right|
$$

(see (3.8) and (3.9) below), and then prescribe for $-2 \pi \leq \arg (z) \leq 2 \pi$

$$
\mathcal{E}_{n, 1}(z)=\sup _{u \in \mathcal{D}(z)}\left\{\left|u^{q-n+1} E_{n-\mu_{1}+\mu_{2}}(u)\right|+\left|u^{q-n} e^{-u}\right|\right\} .
$$

Equation (2.37) defines a real-valued function which is continuous for $-2 \pi \leq \arg (z) \leq 2 \pi$. Our only assumption at this time on the positive integer $n$ is that $n>q$ (and hence $n>\left|\operatorname{Re}\left(\mu_{1}-\mu_{2}\right)\right|$ ). The existence of this supremum then follows from the asymptotic formulas for the generalised exponential integral given above. The asymptotic nature of this function is discussed in $\S 6$ below.

In order to state our main result on error bounds, we must introduce certain terms involved. First, we define

$$
\begin{aligned}
& \gamma_{1}(\delta)= \begin{cases}{[\sin (\delta)]^{-q}} & \left(0<\delta<\frac{1}{2} \pi\right) \\
1 & \left(\delta \geq \frac{1}{2} \pi\right)\end{cases} \\
& \gamma_{2}(\delta)= \begin{cases}{[\sin (\delta)]^{-\left|\operatorname{Re}\left(\mu_{1}-\mu_{2}\right)\right|-q} \exp \left\{(2 \pi-\delta)\left|\operatorname{Im}\left(\mu_{1}-\mu_{2}\right)\right|\right\}} & \left(0<\delta<\frac{1}{2} \pi\right) \\
\exp \left\{\frac{3}{2} \pi\left|\operatorname{Im}\left(\mu_{1}-\mu_{2}\right)\right|\right\} & \left(\delta \geq \frac{1}{2} \pi\right)\end{cases}
\end{aligned}
$$

Next, for each non-zero $z$, we define a path $\mathcal{L}(z)$ in the $t$-plane as follows. When $0 \leq \arg (z) \leq \pi$, the path $\mathcal{L}(z)$ consists of an arc at infinity extending from $t=\infty$ with $\arg (t)=0$ to $t=\operatorname{Re}(z)+i \infty$, and a line parallel to the imaginary axis from $t=\operatorname{Re}(z)+i \infty$ to $t=z$; when $\pi<\arg (z) \leq 2 \pi$, the semi-infinite line runs instead from $t=-|z|+i \infty$ to $t=-|z|$, and in addition the path has the circular component $\mathcal{D}_{2}(z)$. Finally, for $-2 \pi \leq \arg (z)<0$, we define $\mathcal{L}(z)$ to be the conjugate of the path $\mathcal{L}(\bar{z})$, which lies in the sheet $-2 \pi \leq \arg (t)<0$. The path $\mathcal{L}(z)$ is depicted in Figure 2.2 for two values of $\arg (z)$.

There are two crucial properties of $\mathcal{L}(z)$ that we shall use. First, $\left|e^{t}\right| \leq\left|e^{z}\right|$ for all finite $t \in \mathcal{L}(z)$, which is used in deriving (3.7) below. Secondly, on this path $|t| \geq|z|$, and, consequently, the term in square brackets in the bound (2.44) is $O\left(z^{-1}\right)$.

Now, from $(2.26),(2.29)$, and (2.30), we can choose $C_{n, 1}^{(1)}$ (independent of $z$ ) such that

$$
\left|h_{n, 1}^{(1)}(z)\right| \leq C_{n, 1}^{(1)}\left|a_{n, 1} e^{\lambda_{2} z} z^{\mu_{1}-n}\right|\left\{\left|z E_{n-\mu_{1}+\mu_{2}}(z)\right|+\left|e^{-z}\right|\right\}
$$

for $0 \leq \mid \arg (z) \leq \frac{5}{2} \pi$ and $\max \{a, 2 \sigma\}+1 \leq|z|<\infty$. Now, clearly from (2.37),

$$
\left|z E_{n-\mu_{1}+\mu_{2}}(z)\right|+\left|e^{-z}\right| \leq|z|^{n-q} \mathcal{E}_{n, 1}(z),
$$

and hence, from (2.40),

$$
\left|h_{n, 1}^{(1)}(z)\right| \leq C_{n, 1}^{(1)}\left|a_{n, 1} e^{\lambda_{2} z} z^{\mu_{1}-q}\right| \mathcal{E}_{n, 1}(z),
$$




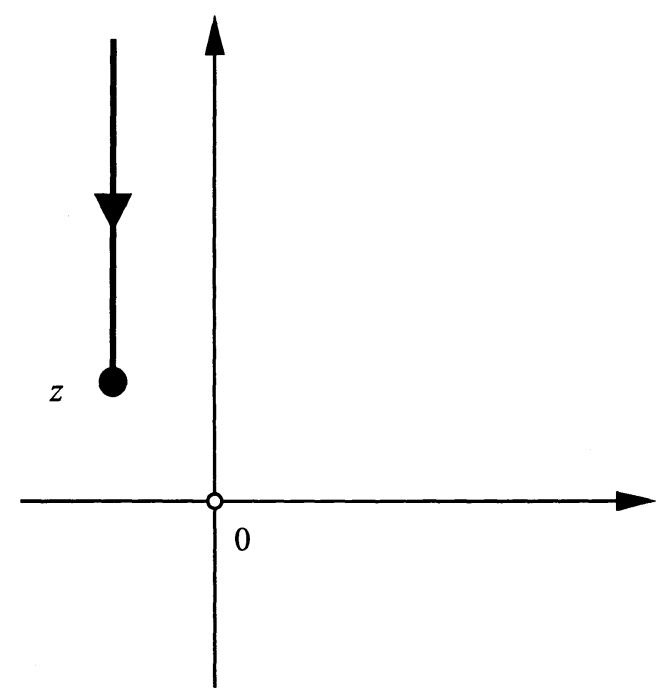

(a)

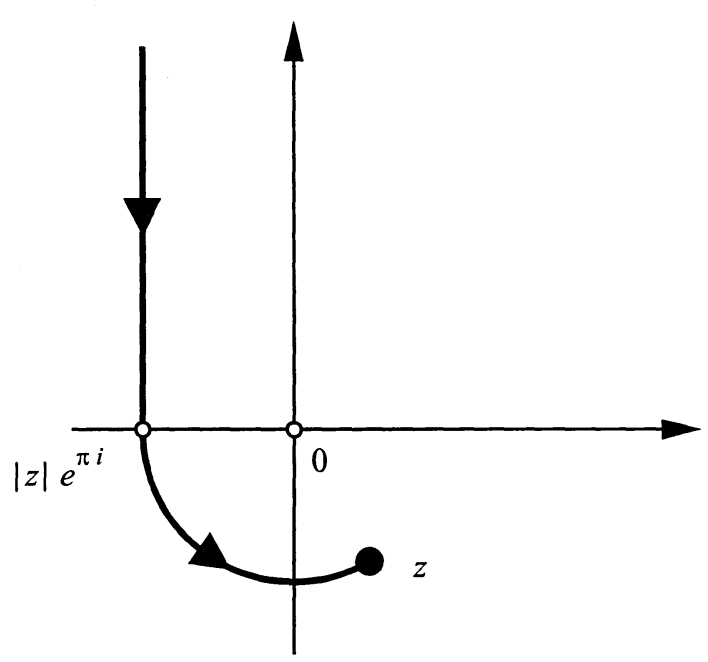

(b)

Figure 2.2. $t$-plane: path $\mathcal{L}(z)$ for: (a) $0<\arg (z)<\pi$, (b) $\pi<\arg (z)<2 \pi$.

which is the inequality we shall use. We prefer to find an appropriate $C_{n, 1}^{(1)}$ to satisfy (2.40) rather than (2.42), since this makes its computation easier. By defining $C_{n, 1}^{(1)}$ via (2.40) for the larger range $0 \leq|\arg (z)| \leq \frac{5}{2} \pi$, and likewise for the more general case in $\S 4$ (see (4.29) below), allows us to use the same constants in $\S 5$.

In the next section, we prove the following theorem.

Theorem 2.2. Select the smallest nonnegative integer $q$ and any positive integer $n$ such that $n>q \geq\left|\operatorname{Re}\left(\mu_{1}-\mu_{2}\right)\right|$. Let $-2 \pi \leq \arg (z) \leq 2 \pi$ and $\max \{a, 2 \sigma\}+1 \leq|z|<$ $\infty$. Then, under the conditions stated in $\S 1$ and in this section, equation (1.1) has the solution

$$
w_{1}(z)=e^{\lambda_{1} z} z^{\mu_{1}} \sum_{s=0}^{n-1} \frac{a_{s, 1}}{z^{s}}+h_{n, 1}^{(1)}(z)+\varepsilon_{n, 1}^{(1)}(z)
$$


where $h_{n, 1}^{(1)}(z)$ is given by (2.26) and

$$
\begin{aligned}
\left|\varepsilon_{n, 1}^{(1)}(z)\right| \leq C_{n, 1}^{(1)} \mid & a_{n, 1} e^{\lambda_{2} z} z^{\mu_{1}-q} \mid \mathcal{E}_{n, 1}(z) \\
\times & {\left[\exp \left\{\int_{\mathcal{L}(z)}\left\{\left|\psi_{1}(t)\right|+\gamma_{2}\left(\frac{1}{2} \pi\right)\left|\psi_{2}(t)\right|\right\}\left|t^{-2} d t\right|\right\}-1\right], }
\end{aligned}
$$

in which

$$
\psi_{j}(t)=\lambda_{j} \tilde{F}(t)-\tilde{F}^{\prime}(t)+\tilde{G}(t)+\left(2+\mu_{j}\right) t^{-1} \tilde{F}(t) .
$$

Remark. If one is considering the cases $0 \leq \arg (z) \leq 2 \pi$ and $-2 \pi \leq \arg (z)<0$ separately, the bound (2.44) (for one of these cases) can be sharpened as follows. If $\operatorname{Im}\left(\mu_{1}-\mu_{2}\right) \arg (z)<0$, then the term $\gamma_{2}\left(\frac{1}{2} \pi\right)$ can be replaced by $\exp \left\{\frac{1}{2} \pi\left|\operatorname{Im}\left(\mu_{1}-\mu_{2}\right)\right|\right\}$; see the comment following Eq. (3.9) below. A similar remark applies to Theorems $2.3,4.3$, and 4.4 .

The corresponding result for $w_{2}(z)$ is as follows. Let $\hat{r}_{n, 2}(z)$ be given by (2.5) with $a_{s, 1}, \mu_{1}, \lambda_{1}$, replaced by $a_{s, 2}, \mu_{2}$, and $\lambda_{2}$, respectively. Then, in place of $(2.20)$ and $(2.21)$, we define coefficients $\left\{\alpha_{n, 2}(k)\right\}_{k=1}^{\infty}$ via the expansion

$$
\begin{aligned}
R_{n, 2}(t) & =\left\{1-\frac{\mu_{2}-\mu_{1}}{t}\right\} \int_{-t}^{\infty} \chi(s)\left\{\frac{n a_{n, 2}}{s^{n+1}}+\frac{\hat{r}_{n, 2}(s)}{s^{n+2}}\right\} d s \\
& =(-1)^{n} \frac{a_{n, 2}}{t^{n}}+(-1)^{n} \sum_{k=1}^{\infty} \frac{\alpha_{n, 2}(k)}{t^{n+k}},
\end{aligned}
$$

and from these, we in turn define

$$
\beta_{n, 2}^{(1)}(s)=\sum_{k=s+1}^{\infty}(-1)^{k} \frac{\alpha_{n, 2}(k)}{a_{n, 2}} \frac{\Gamma\left(n+s+\mu_{1}-\mu_{2}\right)}{\Gamma\left(n+k+\mu_{1}-\mu_{2}\right)} \quad(s=0,1,2, \ldots) .
$$

The approximant for the second solution then is given by

$$
h_{n, 2}^{(1)}(z)=-a_{n, 2} e^{\lambda_{1} z} z^{\mu_{2}-n+1}\left[E_{n+\mu_{1}-\mu_{2}}\left(z e^{-\pi i}\right)\left\{1+\beta_{n, 2}^{(1)}(0)\right\}-\frac{e^{z}}{z} \sum_{s=0}^{\infty} \frac{\beta_{n, 2}^{(1)}(s)}{z^{s}}\right]
$$

and we select $C_{n, 2}^{(1)}$ so that

$$
\left|h_{n, 2}^{(1)}(z)\right| \leq C_{n, 2}^{(1)}\left|a_{n, 2} e^{\lambda_{1} z} z^{\mu_{2}-n}\right|\left\{\left|z E_{n+\mu_{1}-\mu_{2}}\left(z e^{-\pi i}\right)\right|+\left|e^{z}\right|\right\},
$$

uniformly for $0 \leq\left|\arg \left(z e^{-\pi i}\right)\right| \leq \frac{5}{2} \pi$ and $\max \{a, 2 \sigma\}+1 \leq|z|<\infty$. The weight function for the second solution is defined by

$$
\mathcal{E}_{n, 2}(z)=\sup _{u \in \mathcal{D}\left(z e^{-\pi i}\right)}\left\{\left|u^{q-n+1} E_{n+\mu_{1}-\mu_{2}}(u)\right|+\left|u^{q-n} e^{-u}\right|\right\} .
$$

Theorem 2.3. If integers $q$ and $n$ are as in Theorem 2.2, then, under the conditions stated in $§ 1$ and in this section, equation (1.1) has the solution

$$
w_{2}(z)=e^{\lambda_{2} z} z^{\mu_{2}} \sum_{s=0}^{n-1} \frac{a_{s, 2}}{z^{s}}+h_{n, 2}^{(1)}(z)+\varepsilon_{n, 2}^{(1)}(z)
$$


where, for $-\pi \leq \arg (z) \leq 3 \pi$ and $\max \{a, 2 \sigma\}+1 \leq|z|<\infty$,

$$
\begin{aligned}
\left|\varepsilon_{n, 2}^{(1)}(z)\right| \leq C_{n, 2}^{(1)} \mid & a_{n, 2} e^{\lambda_{1} z} z^{\mu_{2}-q} \mid \mathcal{E}_{n, 2}(z) \\
\times & {\left[\exp \left\{\int_{\mathcal{L}\left(z e^{-\pi i}\right)}\left\{\left|\psi_{2}(-t)\right|+\gamma_{2}\left(\frac{1}{2} \pi\right)\left|\psi_{1}(-t)\right|\right\}\left|t^{-2} d t\right|\right\}-1\right] . }
\end{aligned}
$$

We emphasise that we have made no assumption that $n$ be large at this stage; however, for the bounds (2.44) and (2.52) to be asymptotically meaningful, the requirement $n=|z|+O(1)$ will be demonstrated in $\S 6$.

\section{Proof of Theorem 2.2}

We now give details of the proof of Theorem 2.2; the proof of Theorem 2.3 then can be achieved in a similar manner, so it will not be necessary for us to record details. by

By establishing uniform convergence, we show that the solution of (2.13) is given

$$
h_{n, 1}(z)=h_{n, 1}^{(1)}(z)+\sum_{s=1}^{\infty} h_{n, 1}^{(s, 1)}(z)
$$

and we establish the bound (2.44) for $\varepsilon_{n, 1}^{(1)}(z)=\sum_{s=1}^{\infty} h_{n, 1}^{(s, 1)}(z)$ by inductively proving

$$
\left|h_{n, 1}^{(s, 1)}(z)\right| \leq \frac{C_{n, 1}^{(1)}\left|a_{n, 1} e^{\lambda_{2} z} z^{\mu_{1}-q}\right| \mathcal{E}_{n, 1}(z)}{s !}\left[\int_{\mathcal{L}(z)}\left\{\left|\psi_{1}(t)\right|+\gamma_{2}\left(\frac{1}{2} \pi\right)\left|\psi_{2}(t)\right|\right\}\left|t^{-2} d t\right|\right]^{s}
$$

for $s=1,2, \ldots$. To do this, we expand (2.18) into two integrals, and integrate the second by parts to obtain

$$
\begin{aligned}
h_{n, 1}^{(s, 1)}(z)= & \int_{z}^{\infty} K(z, t) \frac{\tilde{G}(t)}{t^{2}} h_{n, 1}^{(s-1,1)}(t) d t-\int_{z}^{\infty} \frac{\partial}{\partial t}\left\{K(z, t) \frac{\tilde{F}(t)}{t^{2}}\right\} h_{n, 1}^{(s-1,1)}(t) d t \\
= & \int_{z}^{\infty} K(z, t)\left\{\frac{t \tilde{G}(t)-t \tilde{F}^{\prime}(t)+2 \tilde{F}(t)}{t^{3}}\right\} h_{n, 1}^{(s-1,1)}(t) d t \\
& -\int_{z}^{\infty} \frac{\partial K(z, t)}{\partial t} \frac{\tilde{F}(t)}{t^{2}} h_{n, 1}^{(s-1,1)}(t) d t .
\end{aligned}
$$

We then expand the kernel $K(z, t)$ and its partial $t$ derivative (see (2.15)), and so express (3.3) in the following form which we shall employ

$$
\begin{array}{r}
h_{n, 1}^{(s, 1)}(z)=e^{\lambda_{1} z} z^{\mu_{1}} \int_{\mathcal{L}(z)} e^{-\lambda_{1} t} t^{-\mu_{1}-2} \psi_{1}(t) h_{n, 1}^{(s-1,1)}(t) d t \\
-e^{\lambda_{2} z} z^{\mu_{2}} \int_{\mathcal{L}(z)} e^{-\lambda_{2} t} t^{-\mu_{2}-2} \psi_{2}(t) h_{n, 1}^{(s-1,1)}(t) d t
\end{array}
$$

where the $\psi_{j}(t)$ are defined by $(2.45)$.

The path of integration from $t=+\infty$ to $t=z$ will, for each value of $s$ in turn, be deformed to the path $\mathcal{L}(z)$ as described in $\S 2$. Justification of the path deformation to $\mathcal{L}(z)$ for each value of $s$ will come from the induction hypothesis (3.2) and from referring to (1.6), (2.26), (2.29), and (2.37).

The main motivation for our definition of $\mathcal{D}(z)$ (for each fixed value of $z$ ) is directly related to $\mathcal{L}(z)$ : for arbitrary $t \in \mathcal{L}(z)$, an important property is $\mathcal{L}(t) \in \mathcal{D}(t) \subset \mathcal{D}(z)$ (in the $u$-plane). The consequence of this will become more apparent in the next paragraph. 
Consider the integrand of the representation (3.4) for $h_{n, 1}^{(1,1)}(z)$ (with $s=1$ and recalling $\left.h_{n, 1}^{(0,1)}(t)=h_{n, 1}^{(1)}(t)\right)$. By employing (2.40) with $z$ replaced by $t$ and noting that

$$
\mathcal{E}_{n, 1}(t)=\sup _{u \in \mathcal{D}(t)}\left\{\left|u^{q-n+1} E_{n-\mu_{1}+\mu_{2}}(u)\right|+\left|u^{q-n} e^{-u}\right|\right\} \leq \mathcal{E}_{n, 1}(z)
$$

(since $\mathcal{D}(t) \subset \mathcal{D}(z)$ for all $t \in \mathcal{L}(z))$, we deduce the inequality

$$
\left|h_{n, 1}^{(1)}(t)\right| \leq C_{n, 1}^{(1)}\left|a_{n, 1} e^{\lambda_{2} t} t^{\mu_{1}-q}\right| \mathcal{E}_{n, 1}(z)
$$

for all $t \in \mathcal{L}(z)$. Thus, using (3.6) in (3.4) results in the bound

$$
\begin{aligned}
\left|h_{n, 1}^{(1,1)}(z)\right| \leq & C_{n, 1}^{(1)}\left|a_{n, 1} e^{\lambda_{2} z} z^{\mu_{1}-q}\right| \mathcal{E}_{n, 1}(z) \\
& \times\left\{\left|z^{q}\right| \int_{\mathcal{L}(z)}\left|\psi_{1}(t) t^{-q-2} d t\right|+\left|z^{\mu_{2}-\mu_{1}+q}\right| \int_{\mathcal{L}(z)}\left|\psi_{2}(t) t^{\mu_{1}-\mu_{2}-q-2} d t\right|\right\} .
\end{aligned}
$$

In deriving this bound, we employed $\left|e^{t}\right| \leq\left|e^{z}\right|$ for all $t \in \mathcal{L}(z)$ in the first integral.

Now, since $q \geq 0$ and $q \geq \operatorname{Re}\left(\mu_{1}-\mu_{2}\right)$, it is straightforward to show for $0 \leq$ $|\arg (z)| \leq 2 \pi$ that

$$
\int_{\mathcal{L}(z)}\left|\psi_{1}(t) t^{-q-2} d t\right| \leq\left|z^{-q}\right| \int_{\mathcal{L}(z)}\left|\psi_{1}(t) t^{-2} d t\right|
$$

and

$$
\int_{\mathcal{L}(z)}\left|\psi_{2}(t) t^{\mu_{1}-\mu_{2}-q-2} d t\right| \leq \gamma_{2}\left(\frac{1}{2} \pi\right)\left|z^{\mu_{1}-\mu_{2}-q}\right| \int_{\mathcal{L}(z)}\left|\psi_{2}(t) t^{-2} d t\right|
$$

where $\gamma_{2}(\delta)$ is defined by $(2.39)$.

Note that if one is restricted to the smaller range $0 \leq \arg (z) \leq 2 \pi$ and $\operatorname{Im}\left(\mu_{1}-\mu_{2}\right)<$ 0 , then the term $\gamma_{2}\left(\frac{1}{2} \pi\right)$ in (3.9) can be replaced by $\exp \left\{\frac{1}{2}\left|\operatorname{Im}\left(\mu_{1}-\mu_{2}\right)\right|\right\}$; a similar replacement holds if $-2 \pi \leq \arg (z)<0$ and $\operatorname{Im}\left(\mu_{1}-\mu_{2}\right)>0$. To prove the first of these assertions, we observe from the definition of $\mathcal{L}(z)$ that if $0 \leq \arg (z) \leq 2 \pi$ and $\operatorname{Im}\left(\mu_{1}-\mu_{2}\right)<0$, then $\pi / 2 \leq \arg (t) \leq \arg (z)$ when $\pi / 2 \leq \arg (z) \leq 2 \pi$, and $\arg (z) \leq \arg (t)<\pi / 2$ when $0 \leq \arg (z) \leq \pi / 2$. Hence, one can show that $\operatorname{Im}\left(\mu_{1}-\right.$ $\left.\mu_{2}\right)\{\arg (z)-\arg (t)\} \leq \frac{1}{2} \pi\left|\operatorname{Im}\left(\mu_{1}-\mu_{2}\right)\right|$ from which the first assertion follows.

Thus, using the inequalities (3.8) and (3.9) in (3.7) yields

$$
\left|h_{n, 1}^{(1,1)}(z)\right| \leq C_{n, 1}^{(1)}\left|a_{n, 1} e^{\lambda_{2} z} z^{\mu_{1}-q}\right| \mathcal{E}_{n, 1}(z) \int_{\mathcal{L}(z)}\left\{\left|\psi_{1}(t)\right|+\gamma_{2}\left(\frac{1}{2} \pi\right)\left|\psi_{2}(t)\right|\right\}\left|t^{-2} d t\right| .
$$

which is what we wanted to prove.

Now let

$$
\Psi(t)=\int_{\mathcal{L}(t)}\left\{\left|\psi_{1}(\nu)\right|+\gamma_{2}\left(\frac{1}{2} \pi\right)\left|\psi_{2}(\nu)\right|\right\}\left|\nu^{-2} d \nu\right|
$$


and suppose that (3.2) holds for some positive $s$. Then, again using (3.4) with $s$ replaced by $s+1$, we find that

$$
\begin{aligned}
\left|h_{n, 1}^{(s+1,1)}(z)\right| \leq & \frac{C_{n, 1}^{(1)}\left|a_{n, 1} e^{\lambda_{2} z} z^{\mu_{1}-q}\right| \mathcal{E}_{n, 1}(z)}{s !} \\
& \times \int_{\mathcal{L}(z)} \Psi^{s}(t)\left\{\left|\psi_{1}(t)\right|+\gamma_{2}\left(\frac{1}{2} \pi\right)\left|\psi_{2}(t)\right|\right\}\left|t^{-2} d t\right| \\
= & \frac{C_{n, 1}^{(1)}\left|a_{n, 1} e^{\lambda_{2} z} z^{\mu_{1}-q}\right| \mathcal{E}_{n, 1}(z)}{s !}\left\{\frac{\Psi^{s+1}(z)}{s+1}\right\}
\end{aligned}
$$

The theorem follows.

\section{Smoothing of Stokes discontinuities: higher approximations}

In this section, we generalise Theorems 2.2 and 2.3 to obtain an exponentially improved asymptotic expansion, again valid for the same ranges of $\arg (z)$, but now with an improved relative error of $O\left(z^{-m}\right)$ for prescribed fixed $m \in \mathbb{N}$. The general procedure for doing this is essentially a modification of the preceding one in $\S 3$. Again, we express $w_{1}(z)$ in the form (2.2) where $h_{n, 1}(z)$ satisfies (2.3). Then, in place of Lemma 2.1 we shall use the following refinement.

Lemma 4.1. Choose a positive integer $m$, and let $A_{j}^{(m)}(z)$ denote the sum to $m$ terms of the asymptotic series in (1.4), i.e.,

$$
A_{j}^{(m)}(z)=\sum_{s=0}^{m-1} \frac{a_{s, j}}{z^{s}} .
$$

Then the functions $e^{\lambda_{1} z} z^{\mu_{1}} A_{1}^{(m)}(z)$ and $e^{\lambda_{2} z} z^{\mu_{2}} A_{2}^{(m)}(z)$ are solutions of the differential equation

$$
\frac{d^{2} w}{d z^{2}}+\tilde{f}^{(m)}(z) \frac{d w}{d z}+\tilde{g}^{(m)}(z) w=0
$$

where

$$
\tilde{f}^{(m)}(z)=f_{0}+\frac{f_{1}}{z}-\frac{d}{d z} \ln \left\{P^{(m)}(z) Q^{(m)}(z)\right\}
$$

and

$$
\begin{aligned}
\tilde{g}^{(m)}(z)=\frac{f_{0}^{2}}{4} & +\frac{f_{0} f_{1}}{2 z}+\frac{f_{1}^{2}-2 f_{1}}{4 z^{2}}-\frac{1}{4}\left\{Q^{(m)}(z)\right\}^{2}-\frac{1}{2}\left(f_{0}+\frac{f_{1}}{z}\right) \frac{d}{d z} \ln \left\{P^{(m)}(z)\right\} \\
& +\left\{P^{(m)}(z)\right\}^{1 / 2} \frac{d^{2}}{d z^{2}}\left[\left\{P^{(m)}(z)\right\}^{-1 / 2}\right] \\
& -\frac{1}{2}\left(f_{0}+\frac{f_{1}}{z}-\frac{d}{d z} \ln \left\{P^{(m)}(z)\right\}\right) \frac{d}{d z} \ln \left\{Q^{(m)}(z)\right\}
\end{aligned}
$$

in which

$$
P^{(m)}(z)=A_{1}^{(m)}(z) A_{2}^{(m)}(z)
$$

and

$$
Q^{(m)}(z)=1+\frac{2 \sigma}{z}-\frac{d}{d z} \ln \left\{\frac{A_{1}^{(m)}(z)}{A_{2}^{(m)}(z)}\right\}
$$


Proof. We seek $\omega(z)$ and $\zeta(z)$ (in general not the same as the corresponding terms in Lemma 2.1, except when $m=1$ ), such that $\omega(z) e^{\lambda_{j} z} z^{\mu_{j}} A_{j}^{(m)}(z)=e^{ \pm \zeta}$. Since these two functions are reciprocals of one another, we immediately deduce that

$$
\omega^{2}(z) e^{\left(\lambda_{1}+\lambda_{2}\right) z} z^{\mu_{1}+\mu_{2}} P^{(m)}(z)=1 .
$$

Solving this then gives $\omega(z)=e^{f_{0} z / 2} z^{f_{1} / 2}\left\{P^{(m)}(z)\right\}^{-1 / 2}$, which in turn yields $\zeta=$ $-\frac{1}{2} z-\sigma \ln (z)+\frac{1}{2} \ln \left\{A_{1}^{(m)}(z)\right\}-\frac{1}{2} \ln \left\{A_{2}^{(m)}(z)\right\}$. The construction of (4.2) now follows in a similar manner to that of $(2.7)$.

An important alternative representation for $\tilde{f}^{(m)}(z)$ and $\tilde{g}^{(m)}(z)$ is given as follows.

Lemma 4.2. Let a number $a^{(m)}$ be chosen such that

$$
\left|P^{(m)}(z)\right|>0
$$

and

$$
\left|Q^{(m)}(z)\right|>0
$$

for $|z|>a^{(m)}$. Then the coefficient functions defined by (4.3) and (4.4) admit convergent expansions of the form

$$
\begin{aligned}
& \tilde{f}^{(m)}(z)=\sum_{s=0}^{\infty} \frac{\tilde{f}_{s}^{(m)}}{z^{s}} \\
& \tilde{g}^{(m)}(z)=\sum_{s=0}^{\infty} \frac{\tilde{g}_{s}^{(m)}}{z^{s}}
\end{aligned}
$$

for $|z|>a^{(m)}$. In (4.9), the first $m+1$ coefficients coincide with those appearing for $f(z)$ (see $(1.2))$, i.e.,

$$
\tilde{f}_{s}^{(m)}=f_{s} \quad(s=0,1,2, \ldots, m)
$$

the next is given by

$$
\begin{aligned}
\tilde{f}_{m+1}^{(m)}=( & \left.m-\mu_{1}\right)\left(m-1-\mu_{1}\right) a_{m-1,1}-\left(m-\mu_{2}\right)\left(m-1-\mu_{2}\right) a_{m-1,2}-2 \sigma f_{m} \\
& +\sum_{k=1}^{m-1}\left\{\lambda_{1} f_{m+1-k}+g_{m+1-k}-\left(k-\mu_{1}\right) f_{m-k}\right\} a_{k, 1} \\
& -\sum_{k=1}^{m-1}\left\{\lambda_{2} f_{m+1-k}+g_{m+1-k}-\left(k-\mu_{2}\right) f_{m-k}\right\} a_{k, 2},
\end{aligned}
$$

with the subsequent ones being given by

$$
\begin{aligned}
\tilde{f}_{s}^{(m)}=- & 2 \sigma \tilde{f}_{s-1}^{(m)}+\sum_{k=1}^{m-1}\left\{\lambda_{1} \tilde{f}_{s-k}^{(m)}+\tilde{g}_{s-k}^{(m)}-\left(k-\mu_{1}\right) \tilde{f}_{s-1-k}^{(m)}\right\} a_{k, 1} \\
& -\sum_{k=1}^{m-1}\left\{\lambda_{2} \tilde{f}_{s-k}^{(m)}+\tilde{g}_{s-k}^{(m)}-\left(k-\mu_{2}\right) \tilde{f}_{s-1-k}^{(m)}\right\} a_{k, 2} \quad(s=m+2, m+3, \ldots) .
\end{aligned}
$$


Similarly, the coefficients appearing in (4.10) are given by

$$
\begin{gathered}
\tilde{g}_{s}^{(m)}=g_{s} \quad(s=0,1,2, \ldots, m) \\
\tilde{g}_{m+1}^{(m)}=-\left(m-\mu_{1}\right)\left(m-1-\mu_{1}\right) a_{m-1,1}-\lambda_{1} \tilde{f}_{m+1}^{(m)}-\mu_{1} f_{m} \\
-\sum_{k=1}^{m-1}\left\{\lambda_{1} f_{m+1-k}+g_{m+1-k}-\left(k-\mu_{1}\right) f_{m-k}\right\} a_{k, 1},
\end{gathered}
$$

and

$$
\begin{array}{r}
\tilde{g}_{s}^{(m)}=-\lambda_{1} \tilde{f}_{s}^{(m)}-\mu_{1} \tilde{f}_{s-1}^{(m)}-\sum_{k=1}^{m-1}\left\{\lambda_{1} \tilde{f}_{s-k}^{(m)}+\tilde{g}_{s-k}^{(m)}-\left(k-\mu_{1}\right) \tilde{f}_{s-1-k}^{(m)}\right\} a_{k, 1} \\
(s=m+2, m+3, \ldots) .
\end{array}
$$

Proof. We first observe that the logarithmic derivative appearing on the right-hand side of (4.6) is $O\left(z^{-2}\right)$ as $z \rightarrow \infty$; hence, from this fact and the definitions (4.1) and (4.5), it is evident that $a^{(m)}$ exists. The analyticity of $\tilde{f}^{(m)}(z)$ and $\tilde{g}^{(m)}(z)$ for $|z|>a^{(m)}$ then follows from the definitions (4.3) and (4.4), together with the fact that in the unbounded annulus neither $P^{(m)}(z)$ nor $Q^{(m)}$ vanishes. Note that when $m=1$, we can choose $a^{(1)}=2 \sigma$.

To obtain the coefficients in the expansions (4.9) and (4.10), we use the fact that asymptotic expansions for the normal solutions of (4.2) are known, namely, they are the exact solutions $e^{\lambda_{j} z} z^{\mu_{j}} A_{j}^{(m)}(z)$ themselves. Thus, if we prescribe

$$
\tilde{a}_{k, j}= \begin{cases}a_{k, j} & (k=0,1,2, \ldots, m-1) \\ 0 & (k=m, m+1, \ldots)\end{cases}
$$

then we can use [8, p. 230, Eq. (1.11)] with $a_{k}=\tilde{a}_{k, j}, f_{k}=\tilde{f}_{k}^{(m)}, g_{k}=\tilde{g}_{k}^{(m)}, \lambda=\lambda_{j}$, and $\mu=\mu_{j}$ for both $j=1$ and $j=2$ where we regard the asymptotic coefficients $\tilde{a}_{k, j}$ as being known and the coefficients $\tilde{f}_{s}^{(m)}$ and $\tilde{g}_{s}^{(m)}$ as unknown. This yields, for each value of $s$, the pair of equations

$$
\begin{aligned}
& \lambda_{j} \tilde{f}_{s}^{(m)}+\tilde{g}_{s}^{(m)}=\left\{\tilde{f}_{0}^{(m)}+2 \lambda_{j}\right\}(s-1) \tilde{a}_{s-1, j}-\mu_{j} \tilde{f}_{s-1}^{(m)} \\
&-\left(s-1-\mu_{j}\right)\left(s-2-\mu_{j}\right) \tilde{a}_{s-2, j} \\
&-\sum_{k=1}^{s-2}\left\{\lambda_{j} \tilde{f}_{s-k}^{(m)}+\tilde{g}_{s-k}^{(m)}-\left(k-\mu_{j}\right) \tilde{f}_{s-1-k}^{(m)}\right\} \tilde{a}_{k, j} \quad \text { for } j=1,2 .
\end{aligned}
$$

To solve for $\tilde{f}_{s}^{(m)}$, subtract (4.18) with $j=2$ from the same with $j=1$, and employ the relations (1.6) and (1.9). Then, on referring to (4.17), one finds that the expression for $\tilde{f}_{s}^{(m)}$ is the same as that for $f_{s}$ when $s=0,1,2, \ldots, m$, and for the subsequent values of $s$, the expressions (4.12) and (4.13) are found. Finally, to determine $\tilde{g}_{s}^{(m)}$, simply set $j=1$ in (4.18) and use (4.11)-(4.13) and (4.17).

Analogous to (2.12), we now re-express (2.3) in the form

$$
\begin{aligned}
h_{n, 1}^{\prime \prime}(z)+ & \tilde{f}^{(m)}(z) h_{n, 1}^{\prime}(z)+\tilde{g}^{(m)}(z) h_{n, 1}(z)=-e^{\lambda_{1} z} z^{\mu_{1}} r_{n, 1}(z) \\
& +\left\{\tilde{g}^{(m)}(z)-g(z)\right\} h_{n, 1}(z)+\left\{\tilde{f}^{(m)}(z)-f(z)\right\} h_{n, 1}^{\prime}(z)
\end{aligned}
$$


so that instead of (2.13), we have the alternative representation

$$
h_{n, 1}(z)=\int_{z}^{\infty} K^{(m)}(z, t)\left\{-e^{\lambda_{1} t} t^{\mu_{1}} \chi^{(m)} r_{n, 1}(t)+\frac{\tilde{G}^{(m)}(t)}{t^{m+1}} h_{n, 1}(t)+\frac{\tilde{F}^{(m)}(t)}{t^{m+1}} h_{n, 1}^{\prime}(t)\right\} d t
$$

where

$$
\begin{gathered}
K^{(m)}(z, t)=A_{1}^{(m)}(z) e^{\lambda_{1} z} z^{\mu_{1}} A_{2}^{(m)}(t) e^{-\lambda_{1} t} t^{-\mu_{1}}-A_{2}^{(m)}(z) e^{\lambda_{2} z} z^{\mu_{2}} A_{1}^{(m)}(t) e^{-\lambda_{2} t} t^{-\mu_{2}} \\
\chi^{(m)}(t)=\left[A_{1}^{(m)}(t) A_{2}^{(m)}(t) Q^{(m)}(t)\right]^{-1}
\end{gathered}
$$

and

$$
\tilde{F}^{(m)}(t)=t^{m+1} \chi^{(m)}(t)\left\{\tilde{f}^{(m)}(t)-f(t)\right\}, \quad \tilde{G}^{(m)}(t)=t^{m+1} \chi^{(m)}(t)\left\{\tilde{g}^{(m)}(t)-g(t)\right\} .
$$

From Lemma 4.2, we observe that $\tilde{F}^{(m)}(t)$ and $\tilde{G}^{(m)}(t)$ are analytic in the unbounded annulus $|t|>\max \left\{a, a^{(m)}\right\}$, and are $O(1)$ as $t \rightarrow \infty$.

On setting $h_{n, 1}(t)$ and $h_{n, 1}^{\prime}(t)$ to zero in the integrand of the right-hand side of (4.20), we obtain as our approximant

$$
\begin{gathered}
h_{n, 1}^{(m)}(z)=A_{2}^{(m)}(z) e^{\lambda_{2} z} z^{\mu_{2}} \int_{z}^{\infty} e^{-t} t^{\mu_{1}-\mu_{2}} A_{1}^{(m)}(t) \chi^{(m)}(t) r_{n, 1}(t) d t \\
-A_{1}^{(m)}(z) e^{\lambda_{1} z} z^{\mu_{1}} \int_{z}^{\infty} A_{2}^{(m)}(t) \chi^{(m)}(t) r_{n, 1}(t) d t
\end{gathered}
$$

which then can be expressed in the form

$$
h_{n, 1}^{(m)}(z)=A_{2}^{(m)}(z) e^{\lambda_{2} z} z^{\mu_{2}} \int_{z}^{\infty} e^{-t} t^{\mu_{1}-\mu_{2}} R_{n, 1}^{(m)}(t) d t
$$

where

$$
R_{n, 1}^{(m)}(t)=-\frac{A_{1}^{(m)}(t) Q^{(m)}(t)}{A_{2}^{(m)}(t)} \int_{t}^{\infty} A_{2}^{(m)}(s) \chi^{(m)}(s) r_{n, 1}(s) d s .
$$

Note that when $m=1$, this expression degenerates to (2.20). Next we expand the function defined by (4.26) in the form

$$
R_{n, 1}^{(m)}(t)=\frac{a_{n, 1}}{t^{n}}+\sum_{k=1}^{\infty} \frac{\alpha_{n, 1}^{(m)}(k)}{t^{n+k}}
$$

which converges for $|t|>\max \left\{a, a^{(m)}\right\}$. The coefficients $\left\{\alpha_{n, 1}^{(m)}(k)\right\}_{k=1}^{\infty}$ depend on $\left\{a_{s, 1}\right\}_{s=1}^{\max \{m, n\}},\left\{a_{s, 2}\right\}_{s=1}^{m},\left\{f_{s}\right\}_{s=0}^{\infty}$, and $\left\{g_{s}\right\}_{s=0}^{\infty}$, and can be computed from (4.26) using (2.4)-(2.6), (4.1), (4.5), and (4.22).

On employing (2.22) and (2.25) in conjunction with (4.25) and (4.27), we obtain our desired expansion

$$
h_{n, 1}^{(m)}(z)=a_{n, 1} A_{2}^{(m)}(z) e^{\lambda_{2} z} z^{\mu_{1}-n+1}\left[E_{n-\mu_{1}+\mu_{2}}(z)\left\{1+\beta_{n, 1}^{(m)}(0)\right\}-\frac{e^{-z}}{z} \sum_{s=0}^{\infty} \frac{\beta_{n, 1}^{(m)}(s)}{z^{s}}\right]
$$


where $\left\{\beta_{n, 1}^{(m)}(s)\right\}$ are defined by (2.27) with $\alpha_{n, 1}(k)$ replaced by $\alpha_{n, 1}^{(m)}(k)$. From (4.28), we deduce the existence of a computable constant $C_{n, 1}^{(m)}$, which is independent of $z$, such that for $0 \leq|\arg (z)| \leq \frac{5}{2} \pi$ and $\max \left\{a, a^{(m)}\right\}+1 \leq|z|<\infty$

$$
\left|h_{n, 1}^{(m)}(z)\right| \leq C_{n, 1}^{(m)}\left|a_{n, 1} e^{\lambda_{2} z} z^{\mu_{1}-n}\right|\left\{\left|z E_{n-\mu_{1}+\mu_{2}}(z)\right|+\left|e^{-z}\right|\right\}
$$

which implies

$$
\left|h_{n, 1}^{(m)}(z)\right| \leq C_{n, 1}^{(m)}\left|a_{n, 1} e^{\lambda_{2} z} z^{\mu_{1}-q}\right| \mathcal{E}_{n, 1}(z) .
$$

In place of (2.18), we define for $s=1,2, \ldots$

$$
h_{n, 1}^{(s, m)}(z)=\int_{z}^{\infty} K^{(m)}(z, t)\left\{\frac{\tilde{G}^{(m)}(t)}{t^{m+1}} h_{n, 1}^{(s-1, m)}(t)+\frac{\tilde{F}^{(m)}(t)}{t^{m+1}} h_{n, 1}^{(s-1, m)^{\prime}}(t)\right\} d t
$$

and then following the procedure which led to (3.4), we arrive at the representation

$$
\begin{aligned}
h_{n, 1}^{(s, m)}(z)=e^{\lambda_{1} z} z^{\mu_{1}} A_{1}^{(m)}(z) \int_{\mathcal{L}(z)} e^{-\lambda_{1} t} t^{-\mu_{1}-m-1} \psi_{1}^{(m)}(t) h_{n, 1}^{(s-1, m)}(t) d t \\
-e^{\lambda_{2} z} z^{\mu_{2}} A_{2}^{(m)}(z) \int_{\mathcal{L}(z)} e^{-\lambda_{2} t} t^{-\mu_{2}-m-1} \psi_{2}^{(m)}(t) h_{n, 1}^{(s-1, m)}(t) d t
\end{aligned}
$$

with

$$
\begin{aligned}
\psi_{j}^{(m)}(t)=A_{3-j}^{(m)}(t)\left\{\lambda_{j} \tilde{F}^{(m)}(t)+\tilde{G}^{(m)}(t)+\left(m+1+\mu_{j}\right) t^{-1} \tilde{F}^{(m)}(t)\right\} \\
-\left\{A_{3-j}^{(m)}(t) \tilde{F}^{(m)}(t)\right\}^{\prime}
\end{aligned}
$$

In order to bound each of the functions successively, we select a positive constant $A^{(m)}$ such that

$$
\left|A_{j}^{(m)}(z)\right| \leq A^{(m)}
$$

for $\max \left\{a, a^{(m)}\right\}+1 \leq|z|<\infty$. Then from (4.33), one can show, under the conditions stated in the proceeding theorem, that for each $s$

$$
\begin{aligned}
\left|h_{n, 1}^{(s, m)}(z)\right| \leq & \frac{C_{n, 1}^{(m)}\left|a_{n, 1} e^{\lambda_{2} z} z^{\mu_{1}-q}\right| \mathcal{E}_{n, 1}(z)}{s !} \\
& \times\left[A^{(m)} \int_{\mathcal{L}(z)}\left\{\left|\psi_{1}^{(m)}(t)\right|+\gamma_{2}\left(\frac{1}{2} \pi\right)\left|\psi_{2}^{(m)}(t)\right|\right\}\left|t^{-m-1} d t\right|\right]^{s} .
\end{aligned}
$$

The proof of the following theorem now follows in a similar manner to that of Theorem 2.2.

Theorem 4.3. Select the smallest nonnegative integer $q$, and any positive integer $n$, such that $n>q \geq\left|\operatorname{Re}\left(\mu_{1}-\mu_{2}\right)\right|$, In addition, select a positive integer $m$, independent of $z$, and let the positive number $a^{(m)}$ be as defined in Lemma 4.2. Let $-2 \pi \leq \arg (z) \leq 2 \pi$ and $\max \left\{a, a^{(m)}\right\}+1 \leq|z|<\infty$. Then under the conditions stated in $\S \S 1-2$, equation (1.1) has the solution

$$
w_{1}(z)=e^{\lambda_{1} z} z^{\mu_{1}} \sum_{s=0}^{n-1} \frac{a_{s, 1}}{z^{s}}+h_{n, 1}^{(m)}(z)+\varepsilon_{n, 1}^{(m)}(z)
$$


where $h_{n, 1}^{(m)}(z)$ is given by (4.28), and

$$
\begin{aligned}
\left|\varepsilon_{n, 1}^{(m)}(z)\right| \leq C_{n, 1}^{(m)}\left|a_{n, 1} e^{\lambda_{2} z} z^{\mu_{1}-q}\right| \mathcal{E}_{n, 1}(z) & \\
\times & {\left[\exp \left\{A^{(m)} \int_{\mathcal{L}(z)}\left\{\left|\psi_{1}^{(m)}(t)\right|+\gamma_{2}\left(\frac{1}{2} \pi\right)\left|\psi_{2}^{(m)}(t)\right|\right\}\left|t^{-m-1} d t\right|\right\}-1\right] . }
\end{aligned}
$$

The corresponding expansion for $w_{2}(z)$ is given as follows. We define $\left\{\alpha_{n, 2}^{(m)}(k)\right\}_{k=1}^{\infty}$ by the expansion

$$
\begin{gathered}
\frac{A_{2}^{(m)}(-t) \tilde{Q}^{(m)}(-t)}{A_{1}^{(m)}(-t)} \int_{-t}^{\infty} A_{1}^{(m)}(s) \chi^{(m)}(s)\left\{\frac{n a_{n, 2}}{s^{n+1}}+\frac{\hat{r}_{n, 2}(s)}{s^{n+2}}\right\} d s \\
=(-1)^{n} \frac{a_{n, 2}}{t^{n}}+(-1)^{n} \sum_{k=1}^{\infty} \frac{\alpha_{n, 2}^{(m)}(k)}{t^{n+k}}
\end{gathered}
$$

where $\tilde{Q}^{(m)}(z)$ is given by (4.6) with the subscripts 1 and 2 interchanged. Then let

$$
\begin{aligned}
h_{n, 2}^{(m)}(z)=- & a_{n, 2} A_{1}^{(m)}(z) e^{\lambda_{1} z} z^{\mu_{2}-n+1} \\
& \times\left[E_{n+\mu_{1}-\mu_{2}}\left(z e^{-\pi i}\right)\left\{1+\beta_{n, 2}^{(m)}(0)\right\}-\frac{e^{z}}{z} \sum_{s=0}^{\infty} \frac{\beta_{n, 2}^{(m)}(s)}{z^{s}}\right],
\end{aligned}
$$

in which $\left\{\beta_{n, 2}^{(m)}(s)\right\}_{s=0}^{\infty}$ are defined by $(2.47)$ with $\alpha_{n, 2}(k)$ replaced by $\alpha_{n, 2}^{(m)}(k)$. Then, if $C_{n, 2}^{(m)}$ is chosen to satisfy $(2.49)$ but with $h_{n, 2}^{(1)}(z)$ replaced by $h_{n, 2}^{(m)}(z)$, we have the following result.

Theorem 4.4. Let $-\pi \leq \arg (z) \leq 3 \pi$ with the other conditions as stated in Theorem 4.3. Then equation (1.1) has the solution

$$
w_{2}(z)=e^{\lambda_{2} z} z^{\mu_{2}} \sum_{s=0}^{n-1} \frac{a_{s, 2}}{z^{s}}+h_{n, 2}^{(m)}(z)+\varepsilon_{n, 2}^{(m)}(z)
$$

where

$$
\begin{aligned}
& \left|\varepsilon_{n, 2}^{(m)}(z)\right| \leq C_{n, 2}^{(m)}\left|a_{n, 2} e^{\lambda_{1} z} z^{\mu_{2}-q}\right| \mathcal{E}_{n, 2}(z) \\
& \times\left[\exp \left\{A^{(m)} \int_{\mathcal{L}\left(z e^{-\pi i}\right)}\left\{\left|\psi_{2}^{(m)}(-t)\right|+\gamma_{2}\left(\frac{1}{2} \pi\right)\left|\psi_{1}^{(m)}(-t)\right|\right\}\left|t^{-m-1} d t\right|\right\}-1\right] .
\end{aligned}
$$

\section{Extensions to other ranges of $\arg (z)$}

We now extend the results derived in Theorems 4.3 and 4.4 to the ranges $2 \pi<$ $|\arg (z)| \leq \frac{5}{2} \pi-\delta$ and $2 \pi<\left|\arg \left(z e^{-\pi i}\right)\right| \leq \frac{5}{2} \pi-\delta$, respectively. We give brief details for the first of these, and there are essentially two modifications required. An appropriate path of integration must be specified for (4.32), and a suitable weight function must be prescribed in place of (2.37). To simplify the details of the extension of Theorem 4.3, we shall assume here that $|z| \leq n+1$ when $2 \pi<\arg (z)<\frac{5}{2} \pi$ with the restrictions on $n$ and $q$ being the same as in $\S \S 2$ and 4 .

For each non-zero $z$ satisfying $\frac{3}{2} \pi<\arg (z)<\frac{5}{2} \pi$, we define a path $\mathcal{L}_{n}(z)$ in the $t$-plane as follows. To do so, we introduce $\hat{z}_{n}=\operatorname{Re}(z)-i \sqrt{(n+1)^{2}-\operatorname{Re}(z)^{2}}$, which is the point satisfying $\frac{3}{2} \pi<\arg \left(\hat{z}_{n}\right)<2 \pi$, having the same real part as $z$, and with modulus $n+1$. The path $\mathcal{L}_{n}(z)$ then consists of an arc at infinity extending from $t=\infty$ with $\arg (t)=0$ to $t=(n+1)+i \infty$, and a line parallel to the imaginary axis 
from $t=-(n+1)+i \infty$ to $t=(n+1) e^{\pi i} ;$ in addition, the path $\mathcal{L}_{n}(z)$ consists of the part of the circular arc $|t|=n+1$ running from $t=(n+1) e^{\pi i}$ to $t=\hat{z}_{n}$, together with the line segment parallel to the imaginary $t$-axis running from $t=\hat{z}_{n}$ to $t=z$. For $-\frac{5}{2} \pi<\arg (z)<-\frac{3}{2} \pi$, we define $\mathcal{L}_{n}(z)$ to be the conjugate of the path $\mathcal{L}_{n}(\bar{z})$, which lies in the sheet $-\frac{5}{2} \pi<\arg (t)<-\frac{3}{2} \pi$. The path $\mathcal{L}_{n}(z)$ is depicted in Figure 5.1 for the case $2 \pi<\arg (z)<\frac{5}{2} \pi$.

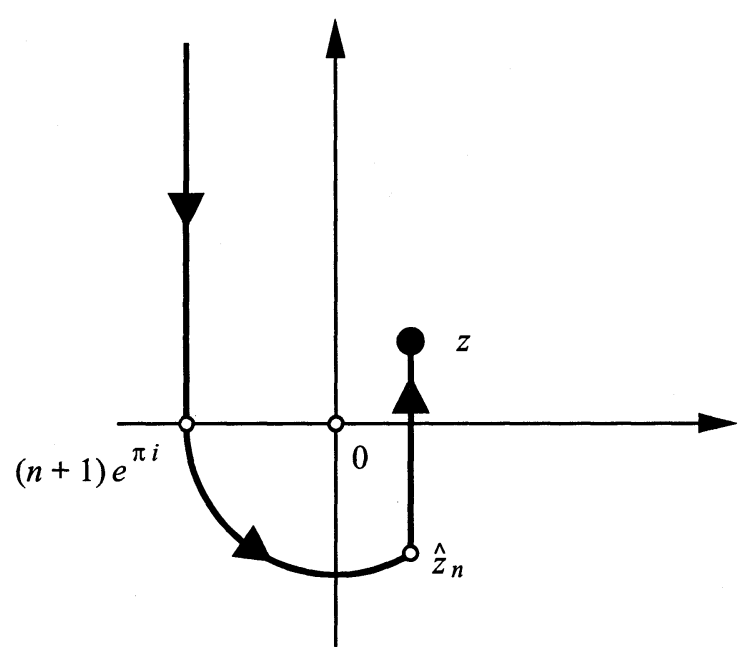

FiguRE 5.1. $t$-plane: path $\mathcal{L}_{n}(z)$ for $2 \pi<\arg (z)<5 \pi / 2$.

We also must define an integration path for each $z^{\prime} \in \mathcal{L}_{n}\left((n+1) e^{ \pm 3 \pi i / 2}\right)$, and this is done simply by defining $\mathcal{L}_{n}\left(z^{\prime}\right)$ to be $\mathcal{L}\left(z^{\prime}\right)$, the segment of $\mathcal{L}_{n}\left((n+1) e^{ \pm 3 \pi i / 2}\right)$ which runs from $t=\infty$ to $t=z^{\prime}$.

As before, $\left|e^{t}\right| \leq\left|e^{z}\right|$ for all finite $t \in \mathcal{L}_{n}(z)$. Also, since $n \geq|z|-1$, on this path $|t| \geq \sin (\delta)|z|$ provided $2 \pi<|\arg (z)| \leq \frac{5}{2} \pi-\delta$. The segment of the path $\mathcal{L}_{n}(z)$ consisting of a semi-infinite straight line and a part of a circle resembles $\mathcal{L}(z)$, except that the circular component of $\mathcal{L}_{n}(z)$ has a fixed radius $n+1$ rather than $|z|$. The importance of this stems from a consideration of all sub-paths $\mathcal{L}_{n}\left(z^{\prime}\right)$ where $z^{\prime} \in \mathcal{L}_{n}(z)$, and we discuss this further below after defining a suitable weight function.

Since the path of integration has been modified, it follows that the weight function, $\hat{\mathcal{E}}_{n, 1}(z)$ say, also must be modified. For $\frac{1}{2} \pi<|\arg (z)|<\frac{5}{2} \pi$, we define it to be

$$
\hat{\mathcal{E}}_{n, 1}(z)=\sup _{u \in \mathcal{L}_{n}(z)}\left\{\left|u^{q-n+1} E_{n-\mu_{1}+\mu_{2}}(u)\right|+\left|u^{q-n} e^{-u}\right|\right\} .
$$

Note that $\hat{\mathcal{E}}_{n, 1}(z)$ varies continuously as $z$ varies continuously in $2 \pi<|\arg (z)|<\frac{5}{2} \pi$. Although in our application $2 \pi<|\arg (z)|<\frac{5}{2} \pi$, we must define $\hat{\mathcal{E}}_{n, 1}(z)$ (and hence $\mathcal{L}_{n}(z)$ ) for the larger range $\frac{1}{2} \pi<|\arg (z)|<\frac{5}{2} \pi$, since $\arg (t)$ lies in this range when $t \in \mathcal{L}_{n}(z)$, and $\hat{\mathcal{E}}_{n, 1}(t)$ is required in each successive approximation.

Let us return to the reason for defining $\mathcal{L}_{n}(z)$ as we did. In bounding $h_{n, 1}^{(s, m)}(z)$ using its representation (4.32) (where $\mathcal{L}(z)$ is now replaced by $\mathcal{L}_{n}(z)$ ), one requires a suitable bound for the integrand, and, in particular, for $h_{n, 1}^{(s-1, m)}\left(z^{\prime}\right)$ for all $z^{\prime} \in \mathcal{L}_{n}(z)$. This in turn requires a suitable bound for $h_{n, 1}^{(s-2, m)}\left(z^{\prime \prime}\right)$ for all $z^{\prime \prime} \in \mathcal{L}_{n}\left(z^{\prime}\right)$ and for all $z^{\prime} \in \mathcal{L}_{n}(z)$, and so on. It is clear from the present choice of $\mathcal{L}_{n}(z)$ that all members of these families of sub-paths are merely sub-paths of $\mathcal{L}_{n}(z)$ itself. However, if $\mathcal{L}_{n}(z)$ 
were defined in a similar manner to $\mathcal{L}(z)$ with a circular component of radius $|z|$, then the following problem would ensue: the point $t=\operatorname{Re}(z) e^{2 \pi i}$ lies on the path $\mathcal{L}_{n}(z)$ whenever $2 \pi<\arg (z)<\frac{5}{2} \pi$, and so in turn, the path $\mathcal{L}_{n}\left(\operatorname{Re}(z) e^{2 \pi i}\right)$ would contain the point $t=\operatorname{Re}(z) e^{\pi i}$. Consequently, the weight function $\hat{\mathcal{E}}_{n, 1}(z)$ would have to be defined as the supremum over a region, $\hat{\mathcal{D}}_{n}(z)$ say, which must contain the point $u=\operatorname{Re}(z) e^{\pi i}$. As a result, this weight function would certainly be bounded below by

$$
\operatorname{Re}(z)^{q-n+1}\left|E_{n-\mu_{1}+\mu_{2}}\left(\operatorname{Re}(z) e^{\pi i}\right)\right| .
$$

When $n=|z|+\alpha$ with $\alpha$ bounded (as will be the case), it then can be shown from (5.2) that the weight function would be asymptotically larger than the approximant $h_{n, 1}^{(m)}(z)$ as $|z| \rightarrow \infty$ (compare (2.29), (2.30), and (4.28)).

With the above definitions, the proof of the following theorem is similar to that of Theorems 2.2 and 4.3 .

Theorem 5.1. Select a positive integer $m$, independent of $z$, and let the positive number $a^{(m)}$ be as defined in Lemma 4.2. Let $2 \pi<|\arg (z)| \leq \frac{5}{2} \pi-\delta$ and $\max \left\{a, a^{(m)}\right\}+$ $1 \leq \operatorname{Re}(z)<\infty$. Choose a constant $\delta>0$, and select the smallest nonnegative integer $q$, and a positive integer $n$, such that $n>q \geq\left|\operatorname{Re}\left(\mu_{1}-\mu_{2}\right)\right|$ and $n \geq|z|-1$. Then, under the conditions stated in $\S \S 1-2$, equation (1.1) has the solution given by (4.36) where

$$
\begin{aligned}
& \left|\varepsilon_{n, 1}^{(m)}(z)\right| \leq C_{n, 1}^{(m)}\left|a_{n, 1} e^{\lambda_{2} z} z^{\mu_{1}-q}\right| \hat{\mathcal{E}}_{n, 1}(z) \\
& \quad \times\left[\exp \left\{A^{(m)} \int_{\mathcal{L}_{n}(z)}\left\{\gamma_{1}(\delta)\left|\psi_{1}^{(m)}(t)\right|+\gamma_{2}(\delta)\left|\psi_{2}^{(m)}(t)\right|\right\}\left|t^{-m-1} d t\right|\right\}-1\right] .
\end{aligned}
$$

Remark. In the derivation of this bound, the following generalisations of (3.8) and (3.9) were used: for $\frac{1}{2} \pi<|\arg (z)| \leq \frac{5}{2} \pi-\delta$, with $|z| \leq n+1$ when $\pi \leq|\arg (z)| \leq \frac{5}{2} \pi-\delta$,

$$
\int_{\mathcal{L}_{n}(z)}\left|\psi_{1}(t) t^{-q-2} d t\right| \leq \gamma_{1}(\delta)\left|z^{-q}\right| \int_{\mathcal{L}_{n}(z)}\left|\psi_{1}(t) t^{-2} d t\right|
$$

and

$$
\int_{\mathcal{L}_{n}(z)}\left|\psi_{2}(t) t^{\mu_{1}-\mu_{2}-q-2} d t\right| \leq \gamma_{2}(\delta)\left|z^{\mu_{1}-\mu_{2}-q}\right| \int_{\mathcal{L}_{n}(z)}\left|\psi_{2}(t) t^{-2} d t\right|
$$

where $\gamma_{j}^{\prime}(\delta)$ are defined by (2.38) and (2.39).

For the second solution, we use the new weight function

$$
\hat{\mathcal{E}}_{n, 2}(z)=\sup _{u \in \mathcal{L}_{n}\left(z e^{-\pi i}\right)}\left\{\left|u^{q-n+1} E_{n+\mu_{1}-\mu_{2}}(u)\right|+\left|u^{q-n} e^{-u}\right|\right\},
$$

and as a result, we have the following extension.

Theorem 5.2. Let $2 \pi<\left|\arg \left(z e^{-\pi i}\right)\right| \leq \frac{5}{2} \pi-\delta$ with the other conditions as stated in Theorem 5.1. Then, equation (1.1) has the solution (4.40) where

$$
\begin{aligned}
& \left|\varepsilon_{n, 2}^{(m)}(z)\right| \leq C_{n, 2}^{(m)}\left|a_{n, 2} e^{\lambda_{1} z} z^{\mu_{2}-q}\right| \hat{\mathcal{E}}_{n, 2}(z) \\
& \quad \times\left[\exp \left\{A^{(m)} \int_{\mathcal{L}_{n}\left(z e^{-\pi i}\right)}\left\{\gamma_{1}(\delta)\left|\psi_{2}^{(m)}(-t)\right|+\gamma_{2}(\delta)\left|\psi_{1}^{(m)}(-t)\right|\right\}\left|t^{-m-1} d t\right|\right\}-1\right] .
\end{aligned}
$$




\section{Asymptotic properties of the expansions}

In this section, we focus on the asymptotic nature of the expansions for $w_{1}(z)$ given by Theorems 4.3 and 5.1. Similar results to those in this section can be obtained regarding the expansions for $w_{2}(z)$. We assume that $0 \leq|\arg (z)| \leq \frac{5}{2} \pi-\delta, \max \left\{a, a^{(m)}\right\}+1 \leq$ $|z|<\infty$, and $m$ is fixed. In addition, we now impose the requirement that

$$
n=|z|+\alpha
$$

where $\alpha$ is bounded. Recall in $\S 5$, we assumed for simplicity that $n \geq|z|-1$, so in that case $\alpha$ also must satisfy $\alpha \geq-1$.

First, to show that the constant in (4.29) can be chosen so that $C_{n, 1}^{(m)}=O(1)$ as $n \rightarrow \infty$, one could select, for instance,

$$
C_{n, 1}^{(m)}=A^{(m)} \max \left\{\left|1+\beta_{n, 1}^{(m)}(0)\right|, \sum_{s=0}^{\infty}\left|\beta_{n, 1}^{(m)}(s)\right|\left[\max \left\{a, a^{(m)}\right\}+1\right]^{-s}\right\}
$$

so that (for fixed $m$ )

$$
C_{n, 1}^{(m)}=A^{(m)}\left\{1+O\left(\frac{a_{n-1,1}}{n a_{n, 1}}\right)\right\} .
$$

From the error bounds given by Theorems 4.3 and 5.1 , it is seen that, as a means of showing that the leading terms provide a meaningful asymptotic approximation to the corresponding exact solutions, it suffices to establish the theorem.

Theorem 6.1. Under the conditions stated in this section

$$
a_{n, 1} e^{\lambda_{2} z} z^{\mu_{1}-q} \mathcal{E}_{n, 1}(z)=O\left\{h_{n, 1}^{(m)}(z)\right\},
$$

uniformly for $-2 \pi \leq \arg (z) \leq 2 \pi$. Furthermore, if $\nu_{0}=0.27846 \cdots$ is the solution of $\ln \left(1 / \nu_{0}\right)=\nu_{0}+1$, and $\delta_{0}=\arcsin \left(\nu_{0}\right)$, then

$$
a_{n, 1} e^{\lambda_{2} z} z^{\mu_{1}-q} \hat{\mathcal{E}}_{n, 1}(z)=O\left\{h_{n, 1}^{(m)}(z)\right\}
$$

uniformly for $2 \pi \leq|\arg (z)| \leq \frac{5}{2} \pi-\delta_{0}$. In degrees, $\delta_{0}=16.2^{\circ}$.

Proof. From the definition (2.37), and the maximum modulus theorem, there exists $\zeta, \zeta^{\prime} \in \partial \mathcal{D}(z)$ such that

$$
\mathcal{E}_{n, 1}(z) \leq\left|\zeta^{q-n+1} E_{n-\mu_{1}+\mu_{2}}(\zeta)\right|+\left|\zeta^{\prime}\right|^{q-n} e^{-\operatorname{Re}\left(\zeta^{\prime}\right)},
$$

when $0 \leq|\arg (z)| \leq 2 \pi ;$ when $2 \pi<|\arg (z)| \leq \frac{5}{2} \pi-\delta, \mathcal{E}_{n, 1}(z)$ is replaced by $\hat{\mathcal{E}}_{n, 1}(z)$ and $\zeta, \zeta^{\prime} \in \mathcal{L}_{n}(z)$.

From now on, we denote generic positive numbers by $K$ and $K^{\prime}$, which can depend on $\delta$ and $m$, but are independent of $z$ (and hence $n$ ). In order to prove (6.5), we aim to show that

$$
\left|h_{n, 1}^{(m)}(z)\right| \geq K\left|a_{n, 1} e^{\lambda_{2} z} z^{\mu_{1}-n+1} E_{n-\mu_{1}+\mu_{2}}(z)\right|
$$

and then

$$
\left|\zeta^{q-n+1} E_{n-\mu_{1}+\mu_{2}}(\zeta)\right|+\left|\zeta^{\prime}\right|^{q-n} e^{-\operatorname{Re}\left(\zeta^{\prime}\right)} \leq K\left|z^{q-n+1} E_{n-\mu_{1}+\mu_{2}}(z)\right| .
$$

From (2.29) and (2.30), we have for $\pi<\arg (\zeta)<3 \pi$ as $\zeta \rightarrow \infty$

$$
\zeta^{-n+1} E_{n-\mu_{1}+\mu_{2}}(\zeta) \sim \frac{2 \pi i e^{\left(n-\mu_{1}+\mu_{2}\right) \pi i} \zeta^{-\mu_{1}+\mu_{2}}}{\Gamma\left(n-\mu_{1}+\mu_{2}\right)}+\frac{\zeta^{-n+1} e^{-\zeta}}{\zeta+n-\mu_{1}+\mu_{2}} .
$$


Thus for large $n$ (so as to employ Stirling's formula) and $|\zeta|$ sufficiently large, we find that

$$
\left|\zeta^{-n+1} E_{n-\mu_{1}+\mu_{2}}(\zeta)\right| \leq K e^{n} n^{-n+1 / 2}\left\{\left(\frac{n}{|\zeta|}\right)^{\operatorname{Re}\left(\mu_{1}-\mu_{2}\right)}+\left(\frac{n}{|\zeta|}\right)^{n} n^{1 / 2} e^{-n-\operatorname{Re}(\zeta)}\right\} .
$$

By examining the asymptotic behaviour of the generalised exponential integral on the negative real axis (see [5, Theorem 3.1]), we find that this bound is actually valid for $\pi \leq \arg (\zeta)<3 \pi$.

For the special case $\zeta=z$ (and recalling that $n=|z|+\alpha$ ), we deduce from (6.9) and Stirling's formula that

$$
\left|E_{n-\mu_{1}+\mu_{2}}(z)\right| \geq K\left|z^{-1 / 2}\right| e^{|z|}
$$

for sufficiently large $|z|$ with $\pi \leq \arg (z)<3 \pi$. Now, to prove (6.7), we use (4.28) to yield

$$
\left|h_{n, 1}^{(m)}(z)\right| \geq\left|a_{n, 1} e^{\lambda_{2} z} z^{\mu_{1}-n+1}\right|\left\{K\left|E_{n-\mu_{1}+\mu_{2}}(z)\right|-K^{\prime}\left|\frac{e^{-z}}{z n}\right|\right\} \text {. }
$$

Therefore, combination of these last two results yields (6.7) for $\pi \leq \arg (z)<3 \pi$. Similarly, the bound can be shown to hold for $-3 \pi<\arg (z) \leq-\pi$. On employing [5, Theorem 3.1], (2.29), and (6.12), we find that (6.7) also holds for $0 \leq|\arg (z)| \leq \pi$.

Next, from [5, Theorem 3.1], (2.29), and (6.11), it is not difficult to show that

$$
\sup _{\zeta^{\prime} \in \partial \mathcal{D}(z)}\left|\zeta^{\prime}\right|^{q-n} e^{-\operatorname{Re}\left(\zeta^{\prime}\right)} \leq K \sup _{\zeta \in \partial \mathcal{D}(z)}\left|\zeta^{q-n+1} E_{n-\mu_{1}+\mu_{2}}(\zeta)\right|
$$

uniformly for $0 \leq|\arg (z)| \leq 2 \pi$. For the corresponding suprema over $\mathcal{L}_{n}(z)$, the same bound can be established from (6.9). Hence, for all $z$ under consideration, the establishment of (6.8) is equivalent to showing that

$$
\left|\zeta^{q-n+1} E_{n-\mu_{1}+\mu_{2}}(\zeta)\right| \leq K\left|z^{q-n+1} E_{n-\mu_{1}+\mu_{2}}(z)\right|,
$$

and to do this, we shall consider 3 cases separately.

Case 1: $0 \leq|\arg (z)| \leq \pi$. In this case, we can exploit the facts that $|\zeta| \geq|z|$ and $\operatorname{Re}(\zeta) \geq \operatorname{Re}(z)$. So to prove (6.14), it suffices to prove

$$
\left|E_{n-\mu_{1}+\mu_{2}}(\zeta)\right| \leq K\left|E_{n-\mu_{1}+\mu_{2}}(z)\right|
$$

and this follows immediately from (2.29) and the stated lower bounds on $|\zeta|$ and $\operatorname{Re}(\zeta)$.

Case 2: $\pi<|\arg (z)| \leq 2 \pi$. Despite this given range of $\arg (z)$, we must bear in mind that $\arg (\zeta)$ can take any value satisfying $0 \leq \mid \arg (\zeta) \leq 2 \pi$. Now $|\zeta| \geq|z|$ if $0 \leq|\arg (\zeta)| \leq \pi$. Thus, in this sub-case, we have from (2.29) and [5, Theorem 3.1]

$$
\left|\zeta^{q-n+1} E_{n-\mu_{1}+\mu_{2}}(\zeta)\right| \leq K|z|^{q-n+1 / 2} e^{|z|} .
$$

Next, when $\pi<|\arg (z)| \leq 2 \pi$, we observe that $|\zeta|=|z|$, so from (6.10), we again arrive at (6.16). Comparing (6.16) with (6.11), we arrive at (6.14) for case 2. 
Case 3: $2 \pi<|\arg (z)| \leq \frac{5}{2}-\delta,|z| \leq n+1$. If $\frac{1}{2} \pi<|\arg (\zeta)| \leq \frac{3}{2} \pi$, then $|\zeta|=n+1 \geq$ $|z|$, and hence (6.14) follows from similar arguments to case 2 . If $\frac{3}{2} \pi<|\arg (\zeta)| \leq$ $\frac{5}{2} \pi-\delta$, then we shall use (6.9) and the bounds

$$
|z| \sin (\delta) \leq \operatorname{Re}(z) \leq|\zeta| \leq n+1
$$

Let $|\zeta|=\nu|z|$ where $\nu$ is bounded, and bounded below by $\sin (\delta)$. Then, from (6.9) and (6.17)

$$
\left|\zeta^{q-n+1} E_{n-\mu_{1}+\mu_{2}}(\zeta)\right| \leq K \max \left\{\frac{n^{q-\mu_{1}+\mu_{2}}}{\Gamma\left(n-\mu_{1}+\mu_{2}\right)},|\nu z|^{q-n} e^{-\nu|z|}\right\} .
$$

If the maximum is attained by the first term in the curly brackets, the analysis follows as before and (6.14) is established. If the second term is the dominant one, then it is seen from (6.11) that a sufficient condition for (6.14) to be established is

$$
|\nu z|^{q-n} e^{-\nu|z|} \leq K|z|^{q-n+1 / 2} e^{|z|} .
$$

If $\nu \geq 1$, then this is indeed satisfied. It remains to consider $\sin (\delta) \leq \nu<1$, and in this case, (6.19) will certainly hold if

$$
\frac{\nu+1}{\ln (1 / \nu)} \geq \frac{n}{|z|}-\frac{\ln |z|}{2|z| \ln (1 / \nu)}
$$

for sufficiently large $|z|$. Since $n=|z|+\alpha$, the right-hand side of (6.20) is bounded above by 1 for sufficiently large $|z|$, and hence we arrive at the sufficient condition $\ln (1 / \nu) \leq \nu+1$, which is equivalent to $\nu \geq \nu_{0}=0.27846 \cdots$. Taking into account (6.17), we summarise that (6.14) will hold for Case 3 if $\delta \geq \arcsin \left(\nu_{0}\right) \approx 16.2^{\circ}$. This completes the proof of Theorem 6.1.

Remark. It is possible to refine Theorem 6.1 to establish a similar bound which is uniformly valid for $0 \leq|\arg (z)| \leq \frac{5}{2} \pi-\delta$ where $\delta>0$ is arbitrarily small. However, this would require a more complicated choice of integration paths and weight functions. This extra complexity would not be worthwhile since, as we indicated in the introduction, Theorem 4.3 and equation (6.4) as they stand, together with appropriate connection formulae, are sufficient to supply error bounds for exponentially-improved asymptotic expansions for all ranges of $\arg (z)$.

\section{Numerical calculation of the coefficients}

As an example on the computation of certain coefficients which appear in this paper, and, in particular, the bounding constants $C_{n, 1}^{(m)}$, consider the equation

$$
\frac{d^{2} w}{d z^{2}}+\frac{1}{z} \frac{d w}{d z}-\frac{1}{4} w=0
$$

This is a form of the modified Bessel equation with the solution corresponding to (2.1) given by

$$
w_{1}(z)=\frac{1}{\sqrt{\pi}} K_{0}\left(\frac{1}{2} z\right) .
$$

One readily finds that in the notation of $\S 1$

$$
\begin{gathered}
\lambda_{1}=-\frac{1}{2}, \quad \lambda_{2}=\frac{1}{2}, \quad \mu_{1}=\mu_{2}=-\frac{1}{2}, \quad a=0, \\
a_{s, 1}=\frac{(-1)^{s}[1 \cdot 3 \cdot 5 \cdots(2 s-1)]^{2}}{4^{s} s !},
\end{gathered}
$$




\begin{tabular}{|c|c|c|}
\hline $\mathrm{s}$ & $\alpha_{20,1}^{(10)}(s) / a_{20,1}$ & $\beta_{20,1}^{(10)}(s-1)$ \\
\hline 1 & -0.2619047621 & 0.01257713459 \\
2 & -0.2383658008 & 0.01036206989 \\
3 & -0.5201157538 & 0.02076233273 \\
4 & -1.712789669 & 0.06334443363 \\
5 & -7.416043231 & 0.2558676955 \\
6 & -39.54852795 & 1.275218536 \\
7 & -250.0219955 & 7.668064527 \\
8 & -1826.788534 & 50.65231777 \\
9 & -15145.85931 & 459.1759540 \\
10 & -65403.09838 & 2288.932597 \\
11 & 29937.79001 & -975.9469163 \\
12 & 24476.09131 & -659.3825135 \\
13 & 140130.4330 & -4035.233399 \\
14 & 432775.7572 & -11002.96409 \\
15 & 2636354.428 & -69677.94197 \\
16 & 11185427.47 & -267304.4003 \\
17 & 71862712.07 & -1829773.461 \\
18 & 255754663.3 & -5990867.516 \\
19 & 1050790920 & -34092565.15 \\
20 & -9516852210 & 244726555.9 \\
\hline
\end{tabular}

TABLE 1

with $a_{s, 2}=(-1)^{s} a_{s, 1}$. From (7.1), we perceive that $R_{n, 1}(t)=a_{n, 1} t^{-n}$, and hence $\beta_{n, 1}(k-1)=\alpha_{n, 1}(k)=0$ for $k=1,2, \ldots$. Hence, for the case $m=1$, we can simply take $C_{n, 1}^{(1)}=1$ in (2.40).

Now consider a higher value of $m$ : for illustrative purposes, let us take $m=10$ and $n=20$. From (4.26), we find the first 20 coefficients that we shall require, as given in Table 1. Next, we calculate a value of $a^{(10)}$. The simplest way of ensuring $\left|A_{j}^{(10)}\right|>0$ for $|z|>\max \left\{a, a^{(10)}\right\}$ is to numerically find positive $\rho$ such that

$$
\sum_{s=1}^{9} \frac{\left|a_{s, 1}\right|}{\rho^{s}}=1
$$

(note that $\left|a_{s, 1}\right|=\left|a_{s, 2}\right|$ ). One finds that $\rho=3.07853 \cdots$, and thus we choose

$$
\max \left\{a, a^{(10)}\right\}=a^{(10)}=3.08 \text {. }
$$

For a bound $A^{(10)}$ corresponding to (4.34), we then use

$$
A^{(10)}=\sum_{s=0}^{9} \frac{\left|a_{s, 1}\right|}{(4.08)^{s}}=1.1794903 \cdots .
$$

Next we find that

$$
\sum_{s=0}^{9} \frac{\left|\beta_{20,1}^{(10)}(s)\right|}{(4.08)^{s}}=0.40316 \cdots
$$


which is smaller than

$$
\left|1+\beta_{20,1}^{(10)}(0)\right|=1.012577 \cdots .
$$

Therefore, in conclusion, we see from (6.2) that the constant we desire can be taken as

$$
C_{20,1}^{(10)}=A^{(10)}\left|1+\beta_{20,1}^{(10)}(0)\right|=1.1943 \cdots .
$$

Remark 1. Due to the choice $m=10$, the coefficients in Table 1 appear quite large. In general, as $m$ increases so does $a^{(m)}$, and hence too the coefficients $\left\{\alpha_{n, j}^{(m)}(k)\right\}_{k=1}^{\infty}$ themselves. However, since $|z|=O(n)$ in applications, the series appearing in (4.28) converge quite rapidly provided $m$ is not too large compared to $n$. For example, one requires 19 terms in the following series to attain the value shown

$$
\sum_{s=0}^{\infty} \frac{\beta_{20,1}^{(10)}(s)}{(10)^{s}}=0.01394215174 \cdots
$$

and in the following, the value shown is attained after taking 10 terms in the given series $n+1$

$$
\sum_{s=0}^{\infty} \frac{\beta_{20,1}^{(10)}(s)}{(20)^{s}}=0.01315724133 \cdots
$$

Remark 2. In practice, one may wish to calculate the coefficients $\left\{\alpha_{n, j}^{(m)}(k)\right\}_{k=0}^{\infty}$ for various values of $n$ (according to the value of $|z|$ under consideration). An efficient way of doing this is to compute one such set of coefficients (for fixed $n$ ), and then use recursion in $n$. For example, consider the coefficients defined by (2.20) and (2.21). By replacing $n$ by $n+1$ in these equations and subtracting the original equations, one finds that

$$
\alpha_{n+1,1}(k)=\alpha_{n, 1}(k+1)-\left[\left\{1-\frac{\mu_{1}-\mu_{2}}{t}\right\} \int_{t}^{\infty} \chi(s)\left\{r_{n+1,1}(s)-r_{n, 1}(s)\right\} d s\right]_{n+k+1}
$$

where $[h(t)]_{k}$ denotes the coefficient of $t^{-k}$ in the expansion of $h(t)$ in inverse powers of $t$. Then, from (2.2) and (2.3), it is not difficult to show that

$$
\begin{aligned}
r_{n+1,1}(z)-r_{n, 1}(z)= & a_{n, 1} z^{-n-2}\left[z^{2}\left(\lambda_{1} f(z)+g(z)+\lambda_{1}^{2}\right)\right. \\
& \left.-z\left(n-\mu_{1}\right)\left(f(z)+2 \lambda_{1}\right)+\left(n-\mu_{1}\right)\left(n-\mu_{1}+1\right)\right] .
\end{aligned}
$$

If we now insert (7.14) into (7.13), we arrive at our desired recursion relation

$$
\alpha_{n+1,1}(k)=\alpha_{n, 1}(k+1)+\frac{a_{n, 1}\left(\mu_{1}-\mu_{2}\right) u_{n, 1}(k)}{n+k}-\frac{a_{n, 1} u_{n, 1}(k+1)}{n+k+1}
$$

where

$$
\begin{gathered}
u_{n, 1}(k)=\left(\mu_{1}-\mu_{2}\right)^{k-1}\left[\lambda_{1}^{2}\left(\mu_{1}-\mu_{2}\right)^{2}-2 \lambda_{1}\left(n-\mu_{1}\right)\left(\mu_{1}-\mu_{2}\right)+\left(n-\mu_{1}\right)\left(n-\mu_{1}+1\right)\right] \\
+\sum_{l=0}^{k+1}\left(\mu_{1}-\mu_{2}\right)^{l}\left[\lambda_{1} f_{k-l+1}-\left(n-\mu_{1}\right) f_{k-l}+g_{k-l+1}\right]
\end{gathered}
$$

in which it is understood that $f_{-1}=0$. Notice that (7.15) and (7.16) simplify considerably in the common case when $\mu_{1}=\mu_{2}$. 
Remark 3. In order to calculate coefficients such as those given by (2.27), one should calculate a starting coefficient (to the desired accuracy) using its convergent series expansion, and then calculate subsequent ones recursively (without having to resort to their convergent expansion representations). For example, from the general form of (2.27), one would use

$$
\beta_{n, 1}^{(m)}(s+1)=-\left(n+s-\mu_{1}+\mu_{2}\right) \beta_{n, 1}^{(m)}(s)-\frac{\alpha_{n, 1}^{(m)}(s+1)}{a_{n, 1}} .
$$

If the coefficients are not decreasing rapidly in absolute value, one would calculate $\beta_{n, 1}^{(m)}(0)$ as the starting coefficient, and then use forward recurrence; otherwise, backward recurrence from some appropriate later starting coefficient may be required.

Acknowledgements. I am grateful to the referee for indicating a number of minor errors in the original manuscript. This research was supported by National Science Foundation grant DMS 9404389.

\section{References}

1. W. Balser, W. B. Jurkat, and D. A. Lutz, On the reduction of connection problems for differential equations with an irregular singular point to ones with only regular singularities, II, SIAM J. Math. Anal. 19 (1988), 398-443.

2. M. V. Berry, Uniform asymptotic smoothing of Stokes' discontinuities, Proc. Roy. Soc. London Ser. A 422 (1989), 7-21.

3. _ Waves near Stokes lines, Proc. Roy. Soc. London Ser. A 427 (1990), 265-280.

4. T. M. Dunster, Asymptotic solutions of second-order linear differential equations having almost coalescent turning points, with an application to the incomplete Gamma function, Proc. Roy. Soc. London Ser. A (1996), (to appear).

5. __ Asymptotics of the generalised exponential integral, and error bounds in the uniform asymptotic smoothing of Stokes' discontinuities, Proc. Roy. Soc. London Ser. A, (1996), (to appear).

6. J. B. McLeod, Smoothing of Stokes discontinuities, Proc. Roy. Soc. London Ser. A 437 (1992), 343-354.

7. A. B. Olde Daalhuis and F. W. J. Olver, Exponentially-improved asymptotic solutions of ordinary differential equations II: irregular singularities of rank one, Proc. Roy. Soc. London Ser. A 445 (1994), 39-56.

8. F. W. J. Olver, Asymptotics and Special Functions, Academic Press, New York, 1974.

9. __ Uniform, exponentially-improved asymptotic expansions for the generalised exponential integral, SIAM J. Math. Anal. 22 (1991), 1460-1489.

10. _ Exponentially-improved asymptotic solutions of ordinary differential equations I: the confluent hypergeometric function, SIAM J. Math. Anal. 24 (1993), 756-767.

11. R. B. Paris, Smoothing of the Stokes phenomenon for high order differential equations, Proc. Roy. Soc. London Ser. A 436 (1992), 165-186.

Department of Mathematical Sciences, San Diego State University, San Diego, Ca 92182 7720 , U.S.A. 